\title{
Stability and Asymptotic Behaviour of Nonlinear Systems: an Introduction
}

H Logemann and E P Ryan

Department of Mathematical Sciences, University of Bath, Bath BA2 7AY, UK

hl@maths.bath.ac.uk epr@maths.bath.ac.uk

\section{Introduction}

To motivate a study of asymptotic behaviour of nonlinear systems modelled by ordinary differential equations and differential inclusions, we indicate how such equations/inclusions arise naturally in control of dynamical process by feedback. The concept of control pertains to modifying the behaviour of the process, by manipulation of inputs to the process, in order to achieve some prescribed goal. Fundamental to this is the notion of feedback: a strategy in which the inputs to the process are determined on the basis of concurrent observations on (or outputs from) the process.

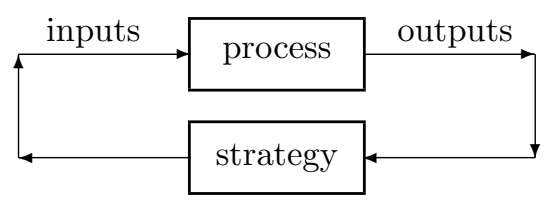

Consider first a finite-dimensional, continuous-time dynamical process, the state of which evolves in $\mathbb{R}^{N}$ and is governed by a controlled ordinary differential equation, with initial data $\left(t_{0}, x^{0}\right)$, of the general form

$$
\dot{x}(t)=g(t, x(t), u(t)), \quad x\left(t_{0}\right)=x^{0},
$$

where the function $u$ is the input or control and the output or observation $y$ is generated via an output map c:

$$
y(t)=c(t, x(t)) .
$$

Under feedback, the input $u(t)$ at time $t$ is determined by the output $y(t)$ via a feedback map $h$ : 


$$
u(t)=h(t, y(t))=h(t, c(t, x(t))) .
$$

Introducing the function $f$ given by $f(t, \xi)=g(t, \xi, h(t, c(t, \xi)))$, we see that the conjunction of (1) and (3) gives rise to an initial-value problem of the following type.

$$
\dot{x}(t)=f(t, x(t)), \quad x\left(t_{0}\right)=x^{0} .
$$

Clearly, in order to ensure that this problem is well posed, the function $f$ is required to be sufficiently regular. In due course, we will make precise the requisite regularity conditions which imply continuity - in their second arguments - of $g, c$ and $h$. Are these continuity conditions reasonable? Perhaps not in the case of the feedback map $h$ - since many feedback strategies are inherently discontinuous, e.g. "bang-bang" or "on-off" control actions. A prototype example is the signum function sgn, which can be embedded in the following set-valued map defined on $\mathbb{R}$ :

$$
x \mapsto \begin{cases}\{+1\}, & x>0 \\ {[-1,+1],} & x=0 . \\ \{-1\}, & x<0\end{cases}
$$

The graph of this map is depicted below.

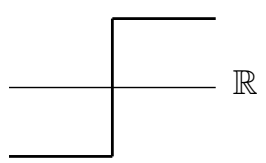

Extrapolating this prototype, we see that situations can arise in which one resorts to a discontinuous feedback which can be embedded in an appropriatelydefined set-valued map $H$ :

$$
u(t) \in H(t, y(t))=H(t, c(t, x(t)) .
$$

Introducing the set-valued map $F$ given by

$$
F(t, \xi)=\{g(t, \xi, u): u \in H(t, c(t, \xi))\},
$$

we see that the conjunction of (1) and (5) gives rise to an initial-value problem for a differential inclusion:

$$
\dot{x}(t) \in F(t, x(t)), \quad x\left(t_{0}\right)=x^{0} .
$$

Again, in order to ensure well-posedness of this problem, $F$ is required to be sufficiently regular (in a sense to be made precise in due course).

The essence of the paper is therefore a study of existence and asymptotic properties of solutions of initial-value problems of the form (4) or (6). Qualitative results on the long-term behaviour of such dynamical processes are of great 
importance in the applications of differential equations, dynamical systems, and control theory to science and engineering. Although Lyapunov's famous memoire on the stability of motion (published in 1892 in Russian) was translated into French in 1907 and reprinted in the USA in 1949 (it was eventually translated into English [13] by A T Fuller in 1992, a hundred years after the publication of the original), it was only at the end of the 1950s that scientists in the West began to appreciate, use, and further develop Lyapunov's seminal contributions to stability theory. This contrasted with the pre-eminence Lyapunov's direct method had achieved in the former Soviet Union as a major mathematical tool in the context of linear and nonlinear stability problems. Today, Lyapunov's direct method is a standard ingredient of the syllabuses of university courses on differential equations, dynamical systems, and control theory taught in mathematics and engineering departments worldwide. With Lyapunov's direct method as exemplar, the paper provides a self-contained and elementary approach to the analysis of certain aspects of the asymptotic behaviour of solutions of ordinary differential equations and differential inclusions. A compendium of results pertaining to asymptotic behaviour of functions is developed. This is achieved by elementary arguments based on concepts of meagreness and weak meagreness which help to capture certain asymptotic properties of functions. This compendium then forms the basis for a unified approach to various results (including generalizations of LaSalle's invariance principle) on asymptotic behaviour of solutions of (nonautonomous) ordinary differential equations and (autonomous) differential inclusions. The material presented in the paper is based on [20] and its precursors [11, 19]. Before embarking on this presentation, we assemble some terminology, notation and background analytical concepts.

\section{Terminology and notation}

Throughout, $\mathbb{N}$ denotes the set of positive integers, and $\mathbb{R}_{+}:=[0, \infty)$. The Euclidean inner product and induced norm on $\mathbb{R}^{N}$ are denoted by $\langle\cdot, \cdot\rangle$ and $\|\cdot\|$, respectively. Let $A$ be a nonempty subset of $\mathbb{R}^{N}$, and let $h: A \rightarrow \mathbb{R}^{P}$. For a subset $U$ of $\mathbb{R}^{P}, h^{-1}(U)$ denotes the preimage of $U$ under $h$, that is, $h^{-1}(U):=\{\xi \in A: h(\xi) \in U\}$; for notational simplicity, if $u \in \mathbb{R}^{P}$, then we write $h^{-1}(u)$ in place of the more cumbersome $h^{-1}(\{u\})$. We recall that $h$ is continuous at a point $\xi_{0} \in A$ if, for every $\varepsilon>0$, there exists $\delta>0$ such that $\left\|h\left(\xi_{0}\right)-h(\xi)\right\| \leq \varepsilon$ for all $\xi$ in $A$ with $\left\|\xi_{0}-\xi\right\| \leq \delta$. If $h$ is continuous at $\xi$ for all $\xi$ in a subset $B$ of $A$, then $h$ is said to be continuous on $B$; if $B=A$, then we simply say that $h$ is continuous. The function $h$ is uniformly continuous on a subset $B$ of $A$ if, for every $\varepsilon>0$, there exists $\delta>0$ such that $\left\|h\left(\xi_{1}\right)-h\left(\xi_{2}\right)\right\| \leq \varepsilon$ for all points $\xi_{1}$ and $\xi_{2}$ of $B$ with $\left\|\xi_{1}-\xi_{2}\right\| \leq \delta$; if $B=A$, then we say that $h$ is uniformly continuous. It is convenient to adopt the convention that $h$ is uniformly continuous on the empty set $\emptyset$. If $h$ is continuous and $B \subset A$ is compact, then $h$ is uniformly continuous on 
$B$. If $h$ is scalar-valued (that is, if $P=1$ ), then $h$ is lower semicontinuous if $\liminf _{\xi^{\prime} \rightarrow \xi} h\left(\xi^{\prime}\right) \geq h(\xi)$ for all $\xi$ in $A$, while $h$ is upper semicontinuous if $-h$ is lower semicontinuous; we remark that $h$ is continuous if, and only if, it is both upper and lower semicontinuous. The Euclidean distance function for a nonempty subset $A \subset \mathbb{R}^{N}$ is the function $d_{A}: \mathbb{R}^{N} \rightarrow \mathbb{R}_{+}$given by $d_{A}(v)=\inf \{\|v-a\|: a \in A\}$. The function $d_{A}$ is globally Lipschitz with Lipschitz constant 1, that is, $\left\|d_{A}(v)-d_{A}(w)\right\| \leq\|v-w\|$ for all $v, w \in \mathbb{R}^{N}$. A function $x: \mathbb{R}_{+} \rightarrow \mathbb{R}^{N}$ is said to approach the set $A$ if $d_{A}(x(t)) \rightarrow 0$ as $t \rightarrow \infty$. For $\varepsilon>0, \mathbb{B}_{\varepsilon}(A):=\left\{\xi \in \mathbb{R}^{N}: d_{A}(\xi)<\varepsilon\right\}$ (the $\varepsilon$-neighbourhood of $A$ ); for $a$ in $\mathbb{R}^{N}$, we write $\mathbb{B}_{\varepsilon}(a)$ in place of $\mathbb{B}_{\varepsilon}(\{a\})$. It is convenient to set $\mathbb{B}_{\varepsilon}(\emptyset)=\emptyset$. The closure of $A$ is denoted by $\operatorname{cl}(A)$.

\section{Background concepts in analysis}

In the context of the real numbers $\mathbb{R}$, Lebesgue measure $\mu$ is a map from a set (the Lebesgue measurable sets) of subsets of $\mathbb{R}$ to the extended non-negative reals $[0, \infty]$. It is an extension of the classical notation of length of an interval in $\mathbb{R}$ to more complicated sets. Whilst there exist subsets of $\mathbb{R}$ which are not measurable, the Lebesgue measurable sets include, for example, all open and closed sets and all sets obtained from these by taking countable unions and intersections. Lebesgue measure has a number of intuitively appealing properties. For example, (i) if $A, B \subset \mathbb{R}$ are measurable sets with $A \subset B$, then $\mu(A) \leq \mu(B)$, (ii) if $A=\cup_{n \in \mathbb{N}} B_{n}$ is a disjoint union of countably many measurable sets, then $\mu(A)=\sum_{n \in \mathbb{N}} \mu\left(B_{n}\right)$, (iii) $\mu$ is translation invariant, that is, if $A \subset \mathbb{R}$ is measurable, then its translation by $b \in \mathbb{R}$, given by $B:=\{a+B: a \in A\}$ is measurable and $\mu(A)=\mu(B)$. The notion of a set of zero (Lebesgue) measure has a simple characterization: $\mu(A)=0$ if, for each $\varepsilon>0$, there exists a countable collection of intervals $I_{n}$ of length $\left|I_{n}\right|$ such that

$$
\sum_{n \in \mathbb{N}}\left|I_{n}\right|<\varepsilon \text { and } \quad A \subset \cup_{n \in \mathbb{N}} I_{n} .
$$

If $A$ is a closed and bounded interval $[a, b]$, then its Lebesgue measure is the length of the interval $\mu(A)=b-a$ and, since each of the sets $\{a\},\{b\}$ and $\{a, b\}$ has measure zero, each of the intervals $(a, b],[a, b)$ and $(a, b)$ also has measure $b-a$.

Let $I \subset \mathbb{R}$ be an interval and $X \subset \mathbb{R}^{N}$ an non-empty set. Two functions $x, y: I \rightarrow X$ are said to be equal almost everywhere (a.e.) if the subset (of $I$ ) on which they differ has zero measure, precisely, if

$$
\{t \in I: x(t) \neq y(t)\} \quad \text { is a set of zero measure. }
$$

Let $x: I \rightarrow X$ and consider a sequence $\left(x_{n}\right)$ of functions $I \rightarrow X$. The sequence $\left(x_{n}\right)$ is said to converge almost everywhere (a.e.) to $x$ if the subset of points $t \in I$ at which $\left(x_{n}(t)\right)$ fails to converge to $x(t)$ has measure zero. A function 
$x: I \rightarrow \mathbb{R}^{N}$ is said to be a measurable function if there exists a sequence $\left(x_{n}\right)$ of piecewise constant functions $I \rightarrow X$ converging almost everywhere to $x$. We remark that the composition $f \circ x$ of a semicontinuous (upper or lower) function $f$ and a measurable function $x$ is a measurable function. A measurable function $x: I \rightarrow X$ is said to be essentially bounded if there exists $K$ such that $\|x(t)\| \leq K$ for almost every (a.e.) $t \in I$ (equivalently, the set of points $t \in I$ at which $\|x(t)\|>K$ has measure zero): the set of such functions $x$ is denoted by $L^{\infty}(I ; X)$. A measurable function $x: I \rightarrow X$ is said to be locally essentially bounded if the restriction of $x$ to every compact (that is, closed and bounded) subset of $I$ is essentially bounded: the set of such functions is denoted by $L_{\mathrm{loc}}^{\infty}(I ; X)$. The (Lebesgue) integral

$$
\int_{I} x(t) d t
$$

of a measurable function $x: I \rightarrow X$ may be defined via the limit of integrals of a suitably chosen sequence of piecewise constant approximants of $x$. The function $x$ is said to be integrable if

$$
\int_{I}\|x(t)\| d t<\infty
$$

The set of such integrable functions $x$ is denoted ${ }^{1}$ by $L^{1}(I ; X)$. The function $x$ is said to be locally integrable if the restriction $\left.x\right|_{J}$ of $x$ to every compact subinterval $J$ of $I$ is integrable (that is, $\int_{J}\|x(t)\| d t<\infty$ ): the set of such functions $x$ is denoted by $L_{\text {loc }}^{1}\left(I ; \mathbb{R}^{N}\right)$. A function $y$ that is the indefinite integral of a locally integrable function $x$ is said to be locally absolutely continuous, that is, a function of the form

$$
t \mapsto y(t)=\int_{c}^{t} x(s) d s
$$

for some $c \in I$ and $x \in L_{\mathrm{loc}}^{1}(I ; X)$; moreover, $y$ is differentiable almost everywhere (a.e.), that is, the set of points $t \in I$ at which the derivative $\dot{y}(t)$ fails to exist has measure zero; furthermore $\dot{y}(t)=x(t)$ for almost all $t \in I$. Thus, one may identify locally absolutely continuous functions as those functions for which the Fundamental Theorem of Calculus holds in the context of Lebesgue integration.

${ }^{1}$ More generally, for $1 \leq p<\infty$, the set of measurable functions $x: I \rightarrow X$ with the property that

$$
\int_{I}\|x(t)\|^{p} d t<\infty
$$

is denoted by $L^{p}\left(I ; \mathbb{R}^{N}\right)$. 


\section{Initial-value problems: existence of solutions}

We proceed to develop a theory of existence of solutions of the initial-value problems (4) and (6): for clarity of exposition, we will assume $t_{0}=0$ in each and, in the context of the latter problem, we restrict attention to the case of an autonomous differential inclusion $\dot{x} \in F(x)$.

\subsection{Ordinary differential equations}

Consider the initial-value problem (4) (with $t_{0}=0$ ), viz.

$$
\dot{x}(t)=f(t, x(t)), \quad x(0)=x^{0} \in \mathbb{R}^{N} .
$$

The ensuing results pertaining to problem (7) can be found in standard texts (see, for example, $[1,8,28,30])$. In order to make sense of the notion solution of (7), it is necessary to impose some regularity on the function $f: \mathbb{R}_{+} \times \mathbb{R}^{N} \rightarrow$ $\mathbb{R}^{N}$. A classical result is: if $f$ is continuous, then (7) has at least one solution, that is, a continuously differentiable function $x: I \rightarrow \mathbb{R}^{N}$, on some non-trivial interval $I$ containing 0 , with $x(0)=x^{0}$ and satisfying the differential equation in (7) for all $t \in I$. However, to insist on continuity of $f$ in its $t$ dependence is difficult to justify (for example, the $t$-dependence of the function $f$ may arise from modelling extraneous disturbances impinging on a dynamical system there is no reason to suppose that such disturbances are continuous). What can we say about existence of solutions in such cases: indeed, how do we even define the concept of solution? Given that we have decided against imposing, on $f$, continuity with respect to its first argument, we have to contend with the possibility of "solutions" of (7) which fail to be continuously differentiable. As a first attempt at arriving at a sensible notion of solution, consider the integrated version of (7):

$$
x(t)=x^{0}+\int_{0}^{t} f(s, x(s)) d s .
$$

We might now consider a solution of (7) to be a function $x:[0, \omega) \rightarrow \mathbb{R}^{N}$ such that (8)) holds for all $t \in[0, \omega)$. For this definition to have substance, the integral on the righthand side must make sense. As outlined in the previous section, the integral does indeed make sense (as a Lebesgue integral) if the integrand $s \mapsto f(s, x(s))$ is a locally integrable function which implies, in particular, that its indefinite integral is locally absolutely continuous. Therefore, we define a (forward) solution of (7) to be a locally absolutely continuous function $x:[0, \omega) \rightarrow \mathbb{R}^{N}, 0<\omega \leq \infty$, such that (8) holds (or, equivalently, such that $x(0)=x^{0}$ and the differential equation in (7) is satisfied for almost all $t \in[0, \omega))$. Consequently, a basic requirement on $f$ is sufficient regularity to ensure that, if $x(\cdot)$ is locally absolutely continuous, then $s \mapsto f(s, x(s))$ is locally integrable. The following hypotheses (usually referred to as the Carathéodory conditions are sufficient for this to hold: 
$(\mathrm{H}):\left\{\begin{array}{l}\text { for each fixed } \xi, f(\cdot, \xi) \text { is a measurable function; } \\ \text { for each fixed } t, f(t, \cdot) \text { is a continuous function; } \\ \text { for each compact } K \subset \mathbb{R}^{N}, \text { there exists a locally integrable function } \\ m \text { such that }\|f(t, \xi)\| \leq m(t) \text { for all }(t, \xi) \in \mathbb{R} \times K .\end{array}\right.$

A solution $x:[0, \omega) \rightarrow \mathbb{R}^{N}$ of $(7)$ is said to be maximal, and $[0, \omega)$ is said to be a maximal interval of existence, if $x$ does not have a right extension that is also a solution. We are now in a position to state the fundamental existence result for the initial-value problem (7).

Theorem 1. Let $f$ satisfy hypothesis $(\mathrm{H})$. Then, for each $x^{0} \in \mathbb{R}^{N},(7)$ has a solution and every solution can be extended to a maximal solution. If $x$ : $[0, \omega) \rightarrow \mathbb{R}^{N}$ is a maximal solution and $\omega<\infty$, then, for every $\tau \in[0, \omega)$ and every compact set $K \subset \mathbb{R}^{N}$, there exists $\sigma \in[\tau, \omega)$ such that $x(\sigma) \notin K$.

This theorem asserts that, under hypothesis $(\mathrm{H})$ and for each $x^{0}$, the initialvalue problem (7) has at least one solution (there may be multiple solutions) and every solution can be maximally extended. By imposing further regularity on $f$, we can infer the existence of precisely one maximal solution (the uniqueness property). Additional regularity sufficient for uniqueness is the following local Lipschitz condition:

$(\mathrm{L}):\left\{\begin{array}{l}\text { for each compact } K \subset \mathbb{R}^{N}, \text { there exists } \lambda \in L_{\text {loc }}^{1}\left(\mathbb{R}_{+} ; \mathbb{R}_{+}\right) \text {such that } \\ \|f(t, \xi)-f(t, \zeta)\| \leq \lambda(t)\|\xi-\zeta\| \text { for all } t \in \mathbb{R}_{+} \text {and all } \xi, \zeta \in K .\end{array}\right.$

Theorem 2. Let $f$ satisfy hypotheses $(\mathrm{H})$ and $(\mathrm{L})$. Then, for each $x^{0} \in \mathbb{R}^{N}$, (7) has a unique maximal solution.

Next, we consider the autonomous counterpart of (7), viz.

$$
\dot{x}(t)=f(x(t)), \quad x(0)=x^{0} \in \mathbb{R}^{N},
$$

where $f: \mathbb{R}^{N} \rightarrow \mathbb{R}^{N}$ is locally Lipschitz:

(La): $\left\{\begin{array}{l}\text { for each compact } K \subset \mathbb{R}^{N}, \text { there exists } \lambda>0 \text { such that } \\ \|f(\xi)-f(\zeta)\| \leq \lambda\|\xi-\zeta\| \text { for all } \xi, \zeta \in K .\end{array}\right.$

In this autonomous setting, we are interested in solutions of (9) in both forwards and backwards time: thus, we deem a continuously differentiable function $x:(\alpha, \omega) \rightarrow \mathbb{R}^{n}$ to be a solution if $0 \in(\alpha, \omega), x(0)=x^{0}$ and the differential equation in (9) holds for all $t \in(\alpha, \omega)$. A solution is maximal if it has no proper left or right extension that is also a solution.

Theorem 3. Let $f$ satisfy hypothesis (La). Then, for each $x^{0} \in \mathbb{R}^{N}$, the autonomous system (9) has unique maximal solution $x:(\alpha, \omega) \rightarrow \mathbb{R}^{N}$.

This theorem implies the existence of a map $\left(t, x^{0}\right) \mapsto \varphi\left(t, x^{0}\right)$ defined by the property that, for each $x^{0}, \varphi\left(\cdot, x^{0}\right)$ is the unique maximal solution $x$ of $(9)$. The domain of $\varphi$ is given by 


$$
\mathcal{D}=\operatorname{dom}(\varphi)=\left\{\left(t, x^{0}\right) \in \mathbb{R} \times \mathbb{R}^{N}: t \in I\left(x^{0}\right)\right\}
$$

where $I\left(x^{0}\right)=(\alpha, \omega)$ denotes the maximal interval of existence of the maximal solution of (9). We refer to $\varphi$ as the flow generated by $f$.

Proposition 1. $\mathcal{D}$ is an open set and $\varphi$ is continuous.

A set $S \subset \mathbb{R}^{N}$ is said to be $\varphi$-invariant or invariant under the flow $\varphi$ if, for every $x^{0} \in S$, the unique maximal solution of (9) has trajectory in $S$.

\subsection{Autonomous differential inclusions}

We now turn attention to the autonomous counterpart of the initial-value problem (6), viz.

$$
\dot{x}(t) \in F(x(t)), \quad x(0)=x^{0} \in \mathbb{R}^{N},
$$

where $\xi \mapsto F(\xi) \subset \mathbb{R}^{N}$ is a set-valued map defined on $\mathbb{R}^{N}$. There is growing literature (see, for example, [2], [6], [7], [10], [12], [27]) pertaining to the study of differential inclusions. By a (forward) solution of (10), we mean a locally absolutely continuous function $x:[0, \omega) \rightarrow \mathbb{R}^{N}, 0<\omega \leq \infty$, with $x(0)=x^{0}$, such that the differential inclusion in (10) is satisfied for almost all $t \in[0, \omega)$. A solution $x:[0, \omega) \rightarrow \mathbb{R}^{N}$ is maximal, and $[0, \omega)$ is a maximal interval of existence, if $x$ has no right extension that is also a solution. We proceed to address the issue of identifying regularity conditions on $F$ sufficient to guarantee the existence of at least one solution of (10). To this end, let $\mathcal{U}$ denote the class of set-valued maps $\xi \mapsto F(\xi) \subset \mathbb{R}^{N}$, defined on $\mathbb{R}^{N}$, that (a) take nonempty convex compact values (that is, for each $\xi \in \mathbb{R}^{N}, F(\xi)$ is a nonempty, convex and compact subset of $\mathbb{R}^{N}$ ) and (b) are upper semicontinuous at each $\xi \in \mathbb{R}^{N}$. A set-valued map $F$ is upper semicontinuous at $\xi \in \mathbb{R}^{N}$ if, for each $\varepsilon>0$, there exists $\delta>0$ such that $F\left(\xi^{\prime}\right) \subset \mathbb{B}_{\varepsilon}(F(\xi))$ for all $\xi^{\prime}$ in $\mathbb{B}_{\delta}(\xi)$, as illustrated below.
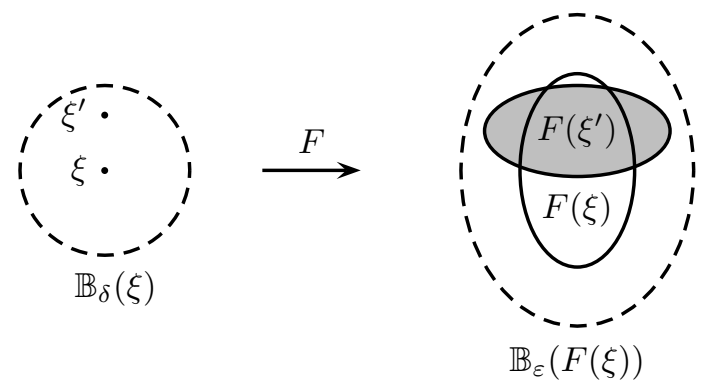

Theorem 4. Let $F \in \mathcal{U}$. For each $x^{0} \in \mathbb{R}^{N}$, (10) has a solution and every solution can be extended to a maximal solution. If $x:[0, \omega) \rightarrow \mathbb{R}^{N}$ is a maximal solution with $\omega<\infty$, then $x$ is unbounded. 
A set $S \subset \mathbb{R}^{N}$ is said to be weakly invariant with respect to the differential inclusion in (10) if, for each $x^{0} \in S$, there exists at least one maximal solution of (10) with trajectory in $S$.

\section{$4.3 \omega$-limit sets}

In his well-known book [4, pp. 197], Birkhoff introduced the notion of an $\omega$-limit point in the context of trajectories of dynamical systems. For the purposes of this paper, it is useful to define the concept of an $\omega$-limit point for arbitrary $\mathbb{R}^{N}$-valued functions defined on $\mathbb{R}_{+}$. Let $x: \mathbb{R}_{+} \rightarrow \mathbb{R}^{N}$. A point $\xi \in \mathbb{R}^{N}$ is an $\omega$-limit point of $x$ if there exists an unbounded sequence $\left(t_{n}\right) \subset \mathbb{R}_{+}$such that $x\left(t_{n}\right) \rightarrow \xi$ as $n \rightarrow \infty$; the (possibly empty) $\omega$-limit set of $x$, denoted by $\Omega(x)$, is the set of all $\omega$-limit points of $x$. The following two lemmas highlight well-known properties of $\omega$-limit sets (see, for example, [1], [12], [14], and [30]).

Lemma 1. The following hold for any function $x: \mathbb{R}_{+} \rightarrow \mathbb{R}^{N}$ :

(a) $\Omega(x)$ is closed.

(b) $\Omega(x)=\emptyset$ if and only if $\|x(t)\| \rightarrow \infty$ as $t \rightarrow \infty$.

(c) If $x$ is continuous and bounded, then $\Omega(x)$ is nonempty, compact, and connected, is approached by $x$, and is the smallest closed set approached by $x$.

(d) If $x$ is continuous and $\Omega(x)$ is nonempty and bounded, then $x$ is bounded and $x$ approaches $\Omega(x)$.

If $x$ happens to be a maximal solution of (9) or of (10), then we can say more.

\section{Lemma 2.}

(a) Let $x: \mathbb{R}_{+} \rightarrow \mathbb{R}^{N}$ be a bounded solution of (9). Then $\Omega(x)$ is nonempty, compact, connected, is approached by $x$, is the smallest closed set approached by $x$, and is invariant under the flow $\varphi$ generated by $f$.

(b) Let $x: \mathbb{R}_{+} \rightarrow \mathbb{R}^{N}$ be a bounded solution of (10). Then $\Omega(x)$ is nonempty, compact, connected, is approached by $x$, is the smallest closed set approached by $x$, and is weakly invariant with respect to the differential inclusion in (10).

\section{Barbălat's lemma, LaSalle's invariance principle, and Lyapunov stability}

A function $y: \mathbb{R}_{+} \rightarrow \mathbb{R}$ is Riemann integrable (on $\mathbb{R}_{+}$) if the improper Riemann integral $\int_{0}^{\infty} y(s) d s$ exists, that is, $y$ is Riemann integrable on $[0, t]$ for

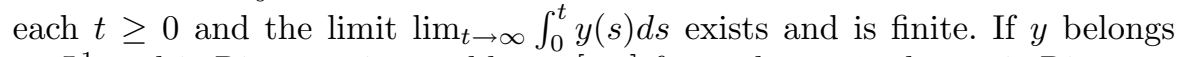
to $L^{1}$ and is Riemann integrable on $[0, t]$ for each $t \geq 0$, then $y$ is Riemann integrable on $\mathbb{R}_{+}$.

First, we highlight the following simple observation, due to Barbălat [3]. 
Lemma 3 (Barbălat's lemma). If $y: \mathbb{R}_{+} \rightarrow \mathbb{R}$ is uniformly continuous and Riemann integrable, then $y(t) \rightarrow 0$ as $t \rightarrow \infty$.

Proof. Suppose to the contrary that $y(t) \nrightarrow 0$ as $t \rightarrow \infty$. Then there exist $\varepsilon>0$ and a sequence $\left(t_{n}\right)$ in $\mathbb{R}_{+}$such that $t_{n+1}-t_{n}>1$ and $\left|y\left(t_{n}\right)\right| \geq \varepsilon$ for all $n$ in $\mathbb{N}$. By the uniform continuity of $y$, there exists $\delta$ in $(0,1)$ such that, for all $n$ in $N$ and all $t$ in $\mathbb{R}_{+}$,

$$
\left|t_{n}-t\right| \leq \delta \quad \Longrightarrow \quad\left|y\left(t_{n}\right)-y(t)\right| \leq \varepsilon / 2 .
$$

Therefore, for all $t$ in $\left[t_{n}, t_{n}+\delta\right]$ and all $n$ in $\mathbb{N},|y(t)| \geq\left|y\left(t_{n}\right)\right|-\left|y\left(t_{n}\right)-y(t)\right| \geq$ $\varepsilon / 2$, from which it follows that

$$
\left|\int_{t_{n}}^{t_{n}+\delta} y(t) d t\right|=\int_{t_{n}}^{t_{n}+\delta}|y(t)| d t \geq \frac{\varepsilon \delta}{2}
$$

for each $n$ in $\mathbb{N}$, contradicting the existence of the improper Riemann integral $\int_{0}^{\infty} y(t) d t$.

Lemma 3 was originally derived in [3] to facilitate the analysis of the asymptotic behaviour of a class of systems of nonlinear second-order equations with forcing. Subsequently, Barbălat's lemma has been widely used in mathematical control theory (see, for example, [9, p. 89], [23, p. 211], and [26, p. 205]).

The following corollary is an immediate consequence of statement (c) of Lemma 1 and Lemma 3.

Corollary 1. Let $G$ be a nonempty closed subset of $\mathbb{R}^{N}$, and let $g: G \rightarrow \mathbb{R}$ be continuous. Assume that $x: \mathbb{R}_{+} \rightarrow \mathbb{R}^{N}$ is bounded and uniformly continuous with $x\left(\mathbb{R}_{+}\right) \subset G$. If $g \circ x$ is Riemann integrable, then $\Omega(x) \subset g^{-1}(0)$ and $x$ approaches $g^{-1}(0)$.

We will use Corollary 1 to derive LaSalle's invariance principle. Let the vector field $f: \mathbb{R}^{N} \rightarrow \mathbb{R}^{N}$ be locally Lipschitz and consider the initial-value problem

$$
\dot{x}=f(x), \quad x(0)=x^{0} \in \mathbb{R}^{N} .
$$

Let $\varphi$ denote the flow generated by $f$, and so $t \mapsto \varphi\left(t, x^{0}\right)$ is the unique solution of (11) defined on its maximal interval of existence $I\left(x^{0}\right)$. If $\mathbb{R}_{+} \subset I\left(x^{0}\right)$ and $\varphi\left(\cdot, x^{0}\right)$ is bounded on $\mathbb{R}_{+}$, then, by assertion (a) of Lemma $2, \Omega\left(\varphi\left(\cdot, x^{0}\right)\right)$ is invariant with respect to the flow $\varphi$.

The following integral-invariance principle provides an intermediate step towards LaSalle's principle and is a consequence of Corollary 1.

Theorem 5 (Integral-invariance principle). Let $G$ be a nonempty closed subset of $\mathbb{R}^{N}$, let $g: G \rightarrow \mathbb{R}$ be continuous, and let $x^{0}$ be a point of $G$. Assume that $\mathbb{R}_{+} \subset I\left(x^{0}\right), \varphi\left(\cdot, x^{0}\right)$ is bounded on $\mathbb{R}_{+}$, and $\varphi\left(\mathbb{R}_{+}, x^{0}\right) \subset G$. If the function $t \mapsto g\left(\varphi\left(t, x^{0}\right)\right)$ is Riemann integrable on $\mathbb{R}_{+}$, then $\varphi\left(\cdot, x^{0}\right)$ approaches the largest invariant subset contained in $g^{-1}(0)$. 
Proof. Since $\varphi\left(\cdot, x^{0}\right)$ is bounded on $\mathbb{R}_{+}$and satisfies the differential equation, it follows that the derivative of $\varphi\left(\cdot, x^{0}\right)$ is bounded on $\mathbb{R}_{+}$. Consequently, $\varphi\left(\cdot, x^{0}\right)$ is uniformly continuous on $\mathbb{R}_{+}$. An application of Corollary 1 together with the invariance property of $\Omega\left(\varphi\left(\cdot, x^{0}\right)\right)$ establishes the claim.

Before proceeding to derive LaSalle's principle, we briefly digress to an example which illustrates that the above integral-invariance principle is of independent interest.

Example 1. Theorem 5 is particularly useful in the context of observed systems. In applications, it is frequently impossible to observe or measure the complete state $x(t)$ of (11) at time $t$. To illustrate the latter comment, consider the observed system given by (11) and the observation

$$
z=c(x),
$$

where $c: \mathbb{R}^{N} \rightarrow \mathbb{R}^{P}$ is continuous with $c(0)=0$.

$$
\dot{x}=f(x), x(0)=x^{0} \stackrel{x}{\longrightarrow} \longrightarrow \longrightarrow z=c(x)
$$

The observation $z$ (also called output or measurement) depends on the state and should be thought of as a quantity that can be observed or measured: an important special case occurring when $z$ is given by one component of the state. Observability concepts relate to the issue of precluding the possibility that different initial states generate the same observation: the initial state of an observable system can, in principle, be recovered from the observation. The system given by (11) and (12) is said to be zero-state observable if the following holds for each $x^{0}$ in $\mathbb{R}^{N}$ :

$$
z(\cdot)=c\left(\varphi\left(\cdot, x^{0}\right)\right)=0 \quad \Longrightarrow \quad \varphi\left(\cdot, x^{0}\right)=0,
$$

that is, the system is zero-state observable if $x(\cdot)=0$ is the only solution generating the zero observation $z(\cdot)=0$. The following corollary of Theorem 5 is contained in [5, Theorem 1.3] and essentially states that, for a zerostate observable system, every bounded trajectory with observation in $L^{p}$ necessarily converges to zero.

Corollary 2. Assume that the observed system given by (11) and (12) is zerostate observable. For given $x^{0}$ in $\mathbb{R}^{N}$ assume that $\mathbb{R}_{+} \subset I\left(x^{0}\right)$ and that $\varphi\left(\cdot, x^{0}\right)$ is bounded on $\mathbb{R}_{+}$. If $\int_{0}^{\infty}\left\|c\left(\varphi\left(t, x^{0}\right)\right)\right\|^{p} d t<\infty$ for some $p$ in $(0, \infty)$, then $\lim _{t \rightarrow \infty} \varphi\left(t, x^{0}\right)=0$.

Proof. By the continuity and boundedness of $\varphi\left(\cdot, x^{0}\right)$, it follows from Lemma 1 that $\varphi\left(\cdot, x^{0}\right)$ approaches its $\omega$-limit set $\Omega:=\Omega\left(\varphi\left(\cdot, x^{0}\right)\right)$ and that $\Omega$ is the smallest closed set approached by $\varphi\left(\cdot, x^{0}\right)$. An application of Proposition 5 with $G=\mathbb{R}^{N}$ and $g(\cdot)=\|c(\cdot)\|^{p}$ shows that $\Omega \subset g^{-1}(0)=c^{-1}(0)$. Let $\xi$ be 
a point of $\Omega$. By the invariance property of $\Omega, \varphi(t, \xi)$ lies in $\Omega$ for all $t$ in $\mathbb{R}$. Consequently, $c(\varphi(\cdot, \xi))=0$. Zero-state observability ensures that $\varphi(\cdot, \xi)=0$, showing that $\xi=0$. Hence $\Omega=\{0\}$, so $\lim _{t \rightarrow \infty} \varphi\left(t, x^{0}\right)=0$.

Theorem 5 is essentially contained in [5, Theorem 1.2]: the proof given therein is not based on Barbălat's lemma. The above proof of Theorem 5 is from [11]. LaSalle's invariance principle (announced in [15], with proof in [16]) is now a straightforward consequence of Theorem 5. For a continuously differentiable function $V: D \subset \mathbb{R}^{N} \rightarrow \mathbb{R}$ (where $D$ is open), it is convenient to define the directional derivative $V_{f}: D \rightarrow \mathbb{R}$ of $V$ in the direction of the vector field $f$ by $V_{f}(\xi)=\langle\nabla V(\xi), f(\xi)\rangle$.

Corollary 3 (LaSalle's invariance principle). Let $D$ be a nonempty open subset of $\mathbb{R}^{N}$, let $V: D \rightarrow \mathbb{R}$ be continuously differentiable, and let $x^{0}$ be a point of D. Assume that $\mathbb{R}_{+} \subset I\left(x^{0}\right)$ and that there exists a compact subset $G$ of $\mathbb{R}^{N}$ such that $\varphi\left(\mathbb{R}_{+}, x^{0}\right) \subset G \subset D$. If $V_{f}(\xi) \leq 0$ for all $\xi$ in $G$, then $\varphi\left(\cdot, x^{0}\right)$ approaches the largest invariant subset contained in $V_{f}^{-1}(0) \cap G$.

Proof. By the compactness of $G$ and the continuity of $V$ on $G$, the function $V$ is bounded on $G$. Combining this with

$$
\int_{0}^{t} V_{f}\left(\varphi\left(s, x^{0}\right)\right) d s=\int_{0}^{t}(d / d s) V\left(\varphi\left(s, x^{0}\right)\right) d s=V\left(\varphi\left(t, x^{0}\right)\right)-V\left(x^{0}\right),
$$

we conclude that the function $t \mapsto \int_{0}^{t} V_{f}\left(\varphi\left(s, x^{0}\right)\right) d s$ is bounded from below: but this function is also nonincreasing (because $V_{f} \leq 0$ on $G$ ) and hence must converge to a finite limit as $t \rightarrow \infty$. Therefore, the function $t \mapsto V_{f}\left(\varphi\left(t, x^{0}\right)\right)$ is Riemann integrable on $\mathbb{R}_{+}$. An application of Theorem 5 (with $g=\left.V_{f}\right|_{G}$ ) completes the proof.

Assume that $f(0)=0$, that is, 0 is an equilibrium of (11). The equilibrium 0 is said to be stable if for every $\varepsilon>0$ there exists $\delta>0$ such that if $\left\|x^{0}\right\| \leq \delta$, then $\mathbb{R}_{+} \subset I\left(x^{0}\right)$ and $\left\|\varphi\left(t, x^{0}\right)\right\| \leq \varepsilon$ for all $t$ in $\mathbb{R}_{+}$. The equilibrium 0 is said to be asymptotically stable if it is stable and there exists $\delta>0$ such that $\left\|\varphi\left(t, x^{0}\right)\right\| \rightarrow 0$ as $t \rightarrow \infty$ for every $x^{0}$ satisfying $\left\|x^{0}\right\| \leq \delta$.

Theorem 6 (Lyapunov's stability theorem). Let $D$ be a nonempty open subset of $\mathbb{R}^{N}$ such that $0 \in D$, and let $V: D \rightarrow \mathbb{R}$ be continuously differentiable with $V(0)=0$. If $V(\xi)>0$ for all $\xi$ in $D \backslash\{0\}$ and $V_{f}(\xi) \leq 0$ for all $\xi$ in $D$, then 0 is a stable equilibrium.

Proof. Let $\varepsilon>0$ be arbitrary. Without loss of generality, we may assume that the closed ball $\overline{\mathbb{B}}_{\varepsilon}(0)$ is contained in $D$. Since the sphere $S_{\varepsilon}:=\{\xi \in$ $\left.\mathbb{R}^{N}:\|\xi\|=\varepsilon\right\}$ is compact and $V$ is continuous and positive-valued on $S_{\varepsilon}$, we see that $V$ achieves a minimum value $m>0$ on $S_{\varepsilon}$, that is,

$$
V(\xi) \geq m \text { for all } \xi \in S_{\varepsilon} \text { and } V(\xi)=m \text { for some } \xi \in S_{\varepsilon} \text {. }
$$


By continuity of the non-negative valued function $V$ and since $V(0)=0$, there exists $\delta \in(0, \varepsilon)$, such that

$$
\|\xi\|<\delta \quad \Longrightarrow \quad V(\xi)<m .
$$

Let $x^{0}$ be such that $\left\|x^{0}\right\|<\delta$. Let $x(\cdot)=\varphi\left(\cdot, x^{0}\right)$ be the maximal solution of (11) with maximal interval of existence $I\left(x^{0}\right)=(\alpha, \omega)$. Seeking a contradiction, suppose $x(\tau) \in S_{\varepsilon}$ for some $\tau \in(0, \omega)$ and assume $\tau$ is the first such time (and so $\|x(t)\|<\varepsilon$ for all $t \in[0, \tau)$ ). Then,

$$
\frac{d}{d t} V(x(t))=V_{f}(x(t)) \leq 0 \quad \forall t \in[0, \tau] .
$$

Therefore, $t \mapsto V(x(t))$ is non-increasing on $[0, \tau]$, whence the contradiction

$$
m \leq V(x(\tau)) \leq V(x(0))=V\left(x^{0}\right)<m .
$$

Therefore, the positive trajectory $x([0, \omega))$ is contained in the closed ball of radius $\varepsilon$ centred at the origin in $\mathbb{R}^{N}$ (and so $\omega=\infty$ ). This completes the proof.

Combining Corollary 3 and Theorem 6, we immediately obtain the following asymptotic stability theorem.

Theorem 7 (Asymptotic stability theorem). Let $D$ be a nonempty open subset of $\mathbb{R}^{N}$ such that $0 \in D$, and let $V: D \rightarrow \mathbb{R}$ be continuously differentiable with $V(0)=0$. If $V(\xi)>0$ for all $\xi$ in $D \backslash\{0\}, V_{f}(\xi) \leq 0$ for all $\xi$ in $D$, and $\{0\}$ is the largest invariant subset of $V_{f}^{-1}(0)$, then 0 is an asymptotically stable equilibrium.

Example 2. In this example, which can also be found in [30], we describe a typical application of Theorem 7 in the context of a general class of nonlinear second-order systems. Consider the system

$$
\ddot{y}(t)+r(y(t), \dot{y}(t))=0, \quad(y(0), \dot{y}(0))=\left(p^{0}, v^{0}\right) \in \mathbb{R}^{2},
$$

where $r: \mathbb{R}^{2} \rightarrow \mathbb{R}$ is locally Lipschitz and differentiable with respect to its second argument. Furthermore, we assume that $r(0,0)=0$. Setting $x(t)=$ $\left(x_{1}(t), x_{2}(t)\right)=(y(t), \dot{y}(t))$, the second-order system (13) can be expressed in the equivalent form (11), where $f: \mathbb{R}_{+} \times \mathbb{R}^{2} \rightarrow \mathbb{R}^{2}$ and $x^{0} \in \mathbb{R}^{2}$ are given by

$$
f(p, v)=(v,-r(p, v)), \quad x^{0}=\left(p^{0}, v^{0}\right) .
$$

Let $\varepsilon>0$, set $D=(-\varepsilon, \varepsilon) \times(-\varepsilon, \varepsilon)$, and define

$$
V: D \rightarrow \mathbb{R}, \quad(p, v) \mapsto \int_{0}^{p} r(s, 0) d s+v^{2} / 2 .
$$

It follows from the mean-value theorem that, for each $(p, v)$ in $D$, there exists a number $\theta=\theta(p, v)$ in the interval $(0,1)$ such that 


$$
V_{f}(p, v)=-v(r(p, v)-r(p, 0))=-v^{2} \frac{\partial r}{\partial v}(p, \theta v) .
$$

Claim. Consider (11) with $f$ and $x^{0}$ given by (14). If $\operatorname{pr}(p, 0)>0$ for all $p$ in $(-\varepsilon, \varepsilon) \backslash\{0\}$ and $(\partial r / \partial v)(p, v)>0$ for all $(p, v)$ in $D$ satisfying $p v \neq 0$, then the equilibrium 0 is asymptotically stable.

We proceed to establish this claim. Using the hypotheses and (15), we infer that $V(p, v)>0$ for all $(p, v)$ in $D \backslash\{0\}$ and $V_{f}(p, v) \leq 0$ for all $(p, v)$ in $D$. Writing $\varphi\left(t, x^{0}\right)=\left(x_{1}(t), x_{2}(t)\right)$, we see that for $x^{0}=\left(p^{0}, 0\right)$ in $D$ with $p^{0} \neq 0, \dot{x}_{2}(0)=-r\left(p^{0}, 0\right) \neq 0$. Similarly, for $x^{0}=\left(0, v^{0}\right)$ in $D$ with $v^{0} \neq 0$, $\dot{x}_{1}(0)=v^{0} \neq 0$. We conclude that solutions with these initial conditions do not remain in $V_{f}^{-1}(0)$, showing that $\{0\}$ is the largest invariant subset of $V_{f}^{-1}(0)$. The claim now follows from Theorem 7 .

As a special case of (13), consider the nonlinear oscillator usually referred to as the Liénard equation

$$
\ddot{y}(t)+d(y(t)) \dot{y}(t)+k(y(t))=0, \quad(y(0), \dot{y}(0))=\left(p^{0}, v^{0}\right) \in \mathbb{R}^{2},
$$

where $d(y) \dot{y}$ represents a friction term that is linear in the velocity and $k(y)$ models a restoring force. We assume that the functions $d: \mathbb{R} \rightarrow \mathbb{R}$ and $k: \mathbb{R} \rightarrow$ $\mathbb{R}$ are locally Lipschitz and $k(0)=0$. It follows from the foregoing discussion on the stability of (13) (with $r$ now given by $r(p, v)=d(p) v+k(p)$ ) that 0 is an asymptotically stable equilibrium state of the Liénard equation, provided that there exists $\varepsilon>0$ such that $p k(p)>0$ and $d(p)>0$ for all $p$ in $(-\varepsilon, \varepsilon)$ with $p \neq 0$.

\section{Generalizations of Barbălat's Lemma}

In Theorems 8 and 9 below, we present generalizations of Barbălat's lemma and of Corollary 1, which will be exploited in subsequent analyses of the behaviour of solutions of non-autonomous differential equations and autonomous differential inclusions. To this end, we introduce the notion of (weak) meagreness that will replace the assumption of Riemann integrability in Barbălat's lemma. The concept of meagreness is defined via the Lebesgue measure $\mu$. However, for the purposes of this tutorial paper, we do not wish to assume familiarity with measure theoretic concepts. As an alternative, we also introduce the notion of weak meagreness (which does not require measure theory).

\section{Definition 1.}

(a) A function $y: \mathbb{R}_{+} \rightarrow \mathbb{R}$ is said to be meagre if $y$ is Lebesgue measurable and $\mu\left(\left\{t \in \mathbb{R}_{+}:|y(t)| \geq \lambda\right\}\right)<\infty$ for all $\lambda>0$.

(b) A function $y: \mathbb{R}_{+} \rightarrow \mathbb{R}$ is said to be weakly meagre if

$$
\lim _{n \rightarrow \infty}\left(\inf _{t \in I_{n}}|y(t)|\right)=0
$$


for every family $\left\{I_{n}: n \in \mathbb{N}\right\}$ of nonempty and pairwise disjoint closed intervals $I_{n}$ in $\mathbb{R}_{+}$with $\inf _{n \in \mathbb{N}}\left|I_{n}\right|>0$, where $\left|I_{n}\right|$ denotes the length of the interval $I_{n}$.

From Definition 1 it follows immediately that a meagre function is weakly meagre. The converse is not true, even in the restricted context of continuous functions. We remark that, if a function $y: \mathbb{R}_{+} \rightarrow \mathbb{R}$ is weakly meagre, then 0 belongs to $\Omega(y)$. The property of (weak) meagreness of a function implies that the function is "close to zero" in some sense: however, it is not the case that (weakly) meagre functions converge to zero as $t \rightarrow \infty$. Indeed, there exist continuous and unbounded functions that are (weakly) meagre: the following is an example of one such function.

Example 3. A continuous unbounded meagre function Consider the continuous unbounded function

$$
\mathbb{R}_{+} \rightarrow \mathbb{R}_{+}, \quad t \mapsto x(t)=\sum_{n \in \mathbb{N}} x_{n}(t)
$$

where, for each $n \in \mathbb{N}, x_{n}$ is continuous and supported on $I_{n}:=\left[n, n+1 / n^{2}\right]$, with graph as shown below.

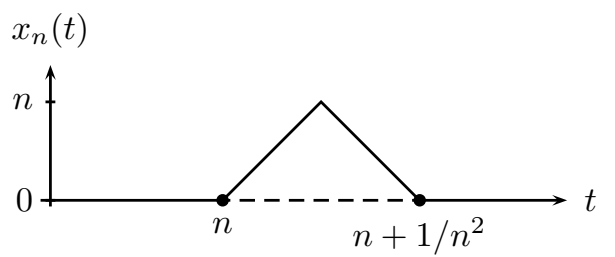

For every $\lambda>0$, the total "length" (measure) of the set $\left\{t \in \mathbb{R}_{+}:|x(t)| \geq \lambda\right\}$ cannot exceed the sum of the lengths $\left|I_{n}\right|=1 / n^{2}$ of the intervals $I_{n}=[n, n+$ $\left.\left(1 / n^{2}\right)\right]$, whence,

$$
\mu\left(\left\{t \in \mathbb{R}_{+}:|x(t)| \geq \lambda\right\}\right) \leq \sum_{n \in \mathbb{N}} \frac{1}{n^{2}}<\infty \quad \forall \lambda>0 .
$$

and so $x$ is meagre (and, a fortiori, weakly meagre).

The above definitions of (weak) meagreness are somewhat obscure: the following result gives more tangible sufficient conditions for meagreness and weak meagreness, respectively.

Proposition 2. Let $y: \mathbb{R}_{+} \rightarrow \mathbb{R}$ be measurable. Then the following statements hold:

(a) If there exists a lower semicontinuous function $\alpha: \mathbb{R}_{+} \rightarrow \mathbb{R}$ such that $\alpha^{-1}(0)=\{0\}, \inf _{s \geq \sigma} \alpha(s)>0$ for all $\sigma>0$, and $\alpha(|y(\cdot)|)$ belongs to $L^{1}$, then $y$ is meagre.

(b) If there exists $\tau>0$ such that $\lim _{t \rightarrow \infty} \int_{t}^{t+\tau}|y(s)| d s=0$, then $y$ is weakly meagre. 
(c) If $y$ is continuous and for every $\delta>0$ there exists $\tau$ in $(0, \delta)$ such that $\int_{t}^{t+\tau} y(s) d s$ converges to 0 as $t \rightarrow \infty$, then $y$ is weakly meagre.

Proof. We prove only part (c) (the proofs of parts (a) and (b) are even more straightforward). Let $y: \mathbb{R}_{+} \rightarrow \mathbb{R}$ be continuous. We show that if $y$ is not weakly meagre, then there exists $\delta>0$ such that for every $\tau$ in $(0, \delta)$ the integral $\int_{t}^{t+\tau} y(s) d s$ does not converge to 0 as $t \rightarrow \infty$. The claim follows then from contraposition. So assume that $y$ is not weakly meagre. Then there exists a family $\left\{I_{n}: n \in \mathbb{N}\right\}$ of nonempty, pairwise disjoint closed intervals with $\delta=\inf _{n \in \mathbb{N}} \mu\left(I_{n}\right)>0$ and a number $\varepsilon>0$ such that $\inf _{t \in I_{n}}|y(t)| \geq \varepsilon$ for each $n$. Since $y$ is continuous, the function $y$ has the same sign on $I_{n}$ for each $n$. Without loss of generality, we may assume that there are infinitely many intervals $I_{n}$ on which $y$ is positive. Then there exists a sequence $\left(n_{k}\right)$ in $\mathbb{N}$ such that $y$ has positive sign on $I_{n_{k}}$ for all $k$. Denoting the left endpoint of $I_{n_{k}}$ by $t_{k}$, we obtain

$$
\int_{t_{k}}^{t_{k}+\tau} y(s) d s \geq \varepsilon \tau>0
$$

for each $k$ in $\mathbb{N}$ and $\tau$ in $(0, \delta)$, showing that the integral $\int_{t}^{t+\tau} y(s) d s$ does not converge to 0 as $t \rightarrow \infty$.

It follows immediately from Proposition 2(a) that, if $y$ belongs to $L^{p}$ for some $p \in[1, \infty)$, then $y$ is meagre (and, a fortior $i$ weakly meagre).

The following result will play a role in the subsequent derivation of generalized versions of Barbălat's lemma.

Lemma 4. Let $A$ and $B$ be nonempty subsets of $\mathbb{R}^{N}$ such that $\operatorname{cl}\left(\mathbb{B}_{\lambda}(B)\right) \subset A$ for some $\lambda>0$. If $x: \mathbb{R}_{+} \rightarrow \mathbb{R}^{N}$ is uniformly continuous on $x^{-1}(A)$, then there exists $\tau>0$ such that

$$
t \in \mathbb{R}_{+}, x(t) \in B \quad \Longrightarrow \quad x(s) \in \mathbb{B}_{\lambda}(B) \quad \forall s \in[t-\tau, t+\tau] \cap \mathbb{R}_{+} .
$$

Proof. Seeking a contradiction, suppose that property (16) does not hold. Then there exist sequences $\left(s_{n}\right)$ and $\left(t_{n}\right)$ in $\mathbb{R}_{+}$such that $x\left(t_{n}\right) \in B$ and $x\left(s_{n}\right) \notin \mathbb{B}_{\lambda}(B)$ for all $n$, and $s_{n}-t_{n} \rightarrow 0$ as $n \rightarrow \infty$. Evidently, $s_{n} \neq t_{n}$ for all $n$. Define $I_{n}$ to be the closed interval with left endpoint $\min \left\{s_{n}, t_{n}\right\}$ and right endpoint $\max \left\{s_{n}, t_{n}\right\}$, and write $T_{n}=\left\{s \in I_{n}: s \notin x^{-1}\left(\mathbb{B}_{\lambda}(B)\right)\right\}$. For each $n$, let $\tau_{n}$ in $T_{n}$ (a compact set) be such that

$$
\left|\tau_{n}-t_{n}\right|=\min _{s \in T_{n}}\left|s-t_{n}\right| .
$$

Clearly, $d_{B}\left(x\left(\tau_{n}\right)\right)=\lambda$ and $d_{B}\left(x\left(t_{n}\right)\right)=0$ for each $n$. Combining this information with the facts that $\tau_{n}$ belongs to $I_{n}$ and $\lim _{n \rightarrow \infty}\left(s_{n}-t_{n}\right)=0$, we conclude that 
(i) ||$x\left(t_{n}\right)-x\left(\tau_{n}\right) \| \geq\left|d_{B}\left(x\left(t_{n}\right)\right)-d_{B}\left(x\left(\tau_{n}\right)\right)\right|=\lambda>0$,

(ii) $t_{n}, \tau_{n} \in x^{-1}(A)$,

(iii) $\left|t_{n}-\tau_{n}\right| \rightarrow 0$ as $n \rightarrow \infty$,

contradicting the hypothesis of the uniform continuity of $x$ on $x^{-1}(A)$. Therefore, property (16) holds.

The following two theorems, the main results of this section, provide our generalizations of Barbălat's lemma.

Theorem 8. Let $G$ be a nonempty closed subset of $\mathbb{R}^{N}$, let $g: G \rightarrow \mathbb{R}$ be a function, and let $x: \mathbb{R}_{+} \rightarrow \mathbb{R}^{N}$ be continuous with $x\left(\mathbb{R}_{+}\right) \subset G$. Assume that each $\xi$ in $G$ for which $g(\xi) \neq 0$ has a neighbourhood $U$ such that

$$
\inf \{|g(w)|: w \in G \cap U\}>0
$$

and $x$ is uniformly continuous on $x^{-1}(U)$. If $g \circ x$ is weakly meagre, then the following statements hold:

(a) $\Omega(x)$ is contained in $g^{-1}(0)$.

(b) If $g^{-1}(0)$ is bounded and $\Omega(x) \neq \emptyset$, then $x$ is bounded and $x$ approaches $g^{-1}(0)$.

(c) If $x$ is bounded, then $g^{-1}(0) \neq \emptyset$ and $x$ approaches $g^{-1}(0)$.

(d) If $x$ is bounded and $g^{-1}(0)$ is totally disconnected, then $\Omega(x)$ consists of a single point $x^{\infty}$ which lies in $g^{-1}(0)$ (in particular, $\left.\lim _{t \rightarrow \infty} x(t)=x^{\infty}\right)$.

Proof. If $\Omega(x)=\emptyset$, then statement (a) holds trivially. Now assume that $\Omega(x) \neq \emptyset$. Let $\xi$ be a point of $\Omega(x)$. Since $G$ is closed and $x\left(\mathbb{R}_{+}\right) \subset G$, $\Omega(x) \subset G$ and thus $\xi$ belongs to $G$. We show that $g(\xi)=0$. Seeking a contradiction, suppose that $g(\xi) \neq 0$. By the hypotheses, there exists a neighbourhood $U$ of $\xi$ such that (17) holds and $x$ is uniformly continuous on $x^{-1}(U)$. Choose $\delta>0$ such that the closure of $\mathbb{B}_{\delta}(\xi)$ lies in $U$. Then

$$
\varepsilon=\inf \left\{|g(w)|: w \in G \cap \mathbb{B}_{\delta}(\xi)\right\}>0 .
$$

Choose $\delta_{1}$ in $(0, \delta)$. Since $\xi$ is an element of $\Omega(x)$, there exists a sequence $\left(t_{n}\right)$ in $\mathbb{R}_{+}$with $t_{n+1}-t_{n}>1$ and $x\left(t_{n}\right)$ in $\mathbb{B}_{\delta_{1}}(\xi)$ for all $n$. An application of Lemma 4 (with $A=U, B=\mathbb{B}_{\delta_{1}}(\xi)$ and $\lambda=\delta-\delta_{1}$ ) shows that there exists $\tau$ in $(0,1)$ such that $x(t)$ is in $\mathbb{B}_{\delta_{2}}(\xi)$ for all $t$ in $\cup_{n \in \mathbb{N}}\left[t_{n}, t_{n}+\tau\right]$. Therefore, by $(18)$,

$$
|(g \circ x)(t)| \geq \varepsilon \quad\left(t \in\left[t_{n}, t_{n}+\tau\right], n \in \mathbb{N}\right) .
$$

Finally, since $t_{n+1}-t_{n}>1$ for all $n$ and $\tau$ belongs to $(0,1)$, the intervals $\left[t_{n}, t_{n}+\tau\right]$ are pairwise disjoint. Combined with (19) this contradicts the weak meagreness of $g \circ x$ and establishes (a). A combination of statement (a) and Lemma 1 yields statements (b)-(d). 
We remark that lower semicontinuity of the function $\xi \mapsto|g(\xi)|$ is sufficient to ensure that (17) holds for some neighbourhood $U$ of any $\xi$ in $G$ with $g(\xi) \neq 0$.

Barbălat's lemma follows immediately from an application of Theorem 8(b) to the situation wherein $N=1, G=\mathbb{R}, g=\mathrm{id}_{\mathbb{R}}$, and $x=y$, in conjunction with the observation that a uniformly continuous and Riemann integrable function $y: \mathbb{R}_{+} \rightarrow \mathbb{R}$ is weakly meagre, implying that 0 is a member of $\Omega(y)$ and thus ensuring that $\Omega(y) \neq \emptyset$. Corollary 1 is a simple consequence of statements (a) and (c) of Theorem 8 .

When compared with Theorem 8, the next result (Theorem 9) posits that $x$ be uniformly continuous on $x^{-1}\left(\mathbb{B}_{\varepsilon}\left(g^{-1}(0)\right)\right)$ for some $\varepsilon>0$. We remark that, in certain situations (for example, if $g^{-1}(0)$ is finite), this assumption is weaker than the uniform continuity assumption imposed on $x$ in Theorem 8. On the other hand, the assumption imposed on $g$ in Theorem 9 is stronger than that in its counterpart in Theorem 8. However, under these modified hypotheses, Theorem 9 guarantees that $x$ approaches $g^{-1}(0) \neq \emptyset$ without assuming the nonemptiness of $\Omega(x)$ or the boundedness of $x$.

Theorem 9. Let $G$ be a nonempty closed subset of $\mathbb{R}^{N}$, and let $g: G \rightarrow \mathbb{R}$ be such that $g^{-1}(0)$ is closed and, for every nonempty closed subset $K$ of $G$,

$$
K \cap g^{-1}(0)=\emptyset \quad \Longrightarrow \quad \inf _{\xi \in K}|g(\xi)|>0 .
$$

Furthermore, let $x: \mathbb{R}_{+} \rightarrow \mathbb{R}^{N}$ be continuous with $x\left(\mathbb{R}_{+}\right) \subset G$. If (i) $x$ is uniformly continuous on $x^{-1}\left(\mathbb{B}_{\varepsilon}\left(g^{-1}(0)\right)\right)$ for some $\varepsilon>0$ and (ii) $g \circ x$ is weakly meagre, then the following statements hold:

(a) $g^{-1}(0) \neq \emptyset, x$ approaches $g^{-1}(0)$, and $\Omega(x)$ is contained in $g^{-1}(0)$.

(b) If $g^{-1}(0)$ is bounded, then $x$ is bounded, $x$ approaches $g^{-1}(0)$, and $\Omega(x)$ is a nonempty subset of $g^{-1}(0)$.

(c) If $g^{-1}(0)$ is bounded and totally disconnected, then $\Omega(x)$ is a singleton $\left\{x^{\infty}\right\}$, where $x^{\infty}$ is a point of $g^{-1}(0)$ (hence, $\lim _{t \rightarrow \infty} x(t)=x^{\infty}$ ).

Proof. For convenience, we set $Z=g^{-1}(0)$. It is clear that $Z \neq \emptyset$ (otherwise, by (20) and the closedness of $G, \gamma=\inf _{\xi \in G}|g(\xi)|>0$ and so $|g(x(t))| \geq \gamma$ for all $t$ in $\mathbb{R}_{+}$, which contradicts the weak meagreness of $\left.g \circ x\right)$. To prove statements (a) and (b), it now suffices to show that $x$ approaches $Z$. From the closedness of $Z$ it then follows immediately that $\Omega(x) \subset Z$; moreover, if $Z$ is bounded, then we can conclude that $x$ is bounded and so $\Omega(x) \neq \emptyset$. Since, by assumption, the trajectory of $x$ is contained in $G$, it is immediate that, if $G=Z$, then $x$ approaches $Z$. Consider the remaining case, wherein $Z$ is a proper subset of $G$. By the closedness of $Z$, there exists $\delta$ in $(0, \varepsilon / 3)$ such that $G \backslash \mathbb{B}_{\delta}(Z) \neq \emptyset$. For $\theta$ in $(0, \delta)$, define

$$
\iota(\theta)=\inf \left\{|g(\xi)|: \xi \in G \backslash \mathbb{B}_{\theta}(Z)\right\}>0,
$$

wherein positivity is a consequence of $(20)$ and the closedness of $G \backslash \mathbb{B}_{\theta}(Z)$. 
Seeking a contradiction, we suppose that $\lim _{t \rightarrow \infty} d_{Z}(x(t)) \neq 0$. Then there exist $\lambda$ in $(0, \delta)$ and a sequence $\left(t_{n}\right)$ in $\mathbb{R}_{+}$with $t_{n} \rightarrow \infty$ as $n \rightarrow \infty$ and $d_{Z}\left(x\left(t_{n}\right)\right) \geq 3 \lambda$ for all $n$. By the weak meagreness of $g \circ x$, there exists a sequence $\left(s_{n}\right)$ in $\mathbb{R}_{+}$with $s_{n} \rightarrow \infty$ as $n \rightarrow \infty$ and $\left|g\left(x\left(s_{n}\right)\right)\right|<\iota(\lambda)$ for all $n$, so $d_{Z}\left(x\left(s_{n}\right)\right) \leq \lambda$ for all $n$. Extracting subsequences of $\left(t_{n}\right)$ and $\left(s_{n}\right)$ (which we do not relabel), we may assume that $s_{n}$ is in $\left(t_{n}, t_{n+1}\right)$ for all $n$. We now have

$$
d_{Z}\left(x\left(t_{n}\right)\right) \geq 3 \lambda, \quad d_{Z}\left(x\left(s_{n}\right)\right) \leq \lambda, \quad s_{n} \in\left(t_{n}, t_{n+1}\right)
$$

for all $n$. By the continuity of $d_{Z} \circ x$, there exists for each $n$ a number $\sigma_{n}$ in $\left(t_{n}, s_{n}\right)$ such that $x\left(\sigma_{n}\right)$ belongs to $B:=\left\{\xi \in G: d_{Z}(\xi)=2 \lambda\right\}$. Extracting a subsequence (which, again, we do not relabel), we may assume that $\sigma_{n+1}-$ $\sigma_{n}>1$ for all $n$. Noting that $\operatorname{cl}\left(\mathbb{B}_{\lambda}(B)\right) \subset \mathbb{B}_{\varepsilon}(Z)$ and invoking Lemma 4 (with $A=\mathbb{B}_{\varepsilon}(Z)$ ), we conclude the existence of $\tau$ in $(0,1)$ such that $d_{Z}(x(t)) \geq \lambda$ for all $t$ in $\left[\sigma_{n}, \sigma_{n}+\tau\right]$ and all $n$. Therefore,

$$
\left\{t \in \mathbb{R}_{+}:|g(x(t))| \geq \iota(\lambda)\right\} \supset \cup_{n \in \mathbb{N}}\left[\sigma_{n}, \sigma_{n}+\tau\right],
$$

which (on noting that the intervals $\left[\sigma_{n}, \sigma_{n}+\tau\right]$, are each of length $\tau>0$ and form a pairwise disjoint family) contradicts the weak meagreness of $g \circ x$. Therefore, $x$ approaches $Z$, implying that statements (a) and (b) hold. Finally, invoking the fact that the $\omega$-limit set of a bounded continuous function is connected, we infer statement (c) from statement (b).

\section{Nonautonomous ordinary differential equations}

Consider again the initial-value problem (7) for a nonautonomous ordinary differential equation

$$
\dot{x}(t)=f(t, x(t)), \quad x(0)=x^{0} \in \mathbb{R}^{N},
$$

where $f: \mathbb{R}_{+} \times \mathbb{R}^{N} \rightarrow \mathbb{R}^{N}$ is a Carathéodory function, that is, $f$ satisfies hypothesis $(\mathrm{H})$. Recall that, by Theorem 1 , for each $x^{0} \in \mathbb{R}^{N},(21)$ has at least one solution and every solution can be extended to a maximal solution. With a view to highlighting a particular subclass of Carathéodory functions $f$, we introduce the notion of uniform local integrability.

Definition 2. A function $m: \mathbb{R}_{+} \rightarrow \mathbb{R}$ is uniformly locally integrable if $m$ belongs to $L_{l o c}^{1}$ and if for each $\varepsilon>0$ there exists $\tau>0$ such that

$$
\int_{t}^{t+\tau}|m(s)| d s \leq \varepsilon
$$

for all $t$ in $\mathbb{R}_{+}$. 
Clearly, a locally integrable function $m: \mathbb{R}_{+} \rightarrow \mathbb{R}$ is uniformly locally integrable if, and only if, the function $t \mapsto \int_{0}^{t}|m(s)| d s$ is uniformly continuous. It is readily verified that, if $m$ belongs to $L^{p}$ for some $p(1 \leq p \leq \infty)$, then $m$ is uniformly locally integrable. We now introduce a particular subclass of Carathéodory functions.

Definition 3. For a nonempty subset $A$ of $\mathbb{R}^{N}, \mathcal{F}(A)$ denotes the class of Carathéodory functions $f: \mathbb{R}_{+} \times \mathbb{R}^{N} \rightarrow \mathbb{R}^{N}$ with the property that there exists a uniformly locally integrable function $m$ such that $\|f(t, \xi)\| \leq m(t)$ for all $(t, \xi)$ in $\mathbb{R}_{+} \times A$.

The next proposition shows that under suitable uniform local integrability assumptions relating to $f$, solutions of (21) satisfy the uniform continuity assumptions required for an application of Theorems 8 and 9 .

Proposition 3. Let $A$ and $B$ be nonempty subsets of $\mathbb{R}^{N}$ with the property that $\mathbb{B}_{\varepsilon}(A) \cap B \neq \emptyset$ for some $\varepsilon>0$, and let $f$ belong to $\mathcal{F}\left(\mathbb{B}_{\varepsilon}(A) \cap B\right)$. If $x: \mathbb{R}_{+} \rightarrow \mathbb{R}^{N}$ is a global solution of (21) such that $x\left(\mathbb{R}_{+}\right) \subset B$, then $x$ is uniformly continuous on $x^{-1}(A)$.

Proof. If $x^{-1}(A)=\emptyset$, then the claim holds trivially. Assume that $x^{-1}(A) \neq \emptyset$. Since $f$ belongs to $\mathcal{F}\left(\mathbb{B}_{\varepsilon}(A) \cap B\right)$, there exists a uniformly locally integrable function $m$ such that $\|f(t, w)\| \leq m(t)$ for all $(t, w)$ in $\mathbb{R}_{+} \times\left(\mathbb{B}_{\varepsilon}(A) \cap B\right)$. Let $\delta$ in $(0, \varepsilon)$ be arbitrary. Choose $\tau>0$ such that $\int_{t}^{t+\tau} m \leq \delta$ for all $t$ in $\mathbb{R}_{+}$. Let $t_{1}$ and $t_{2}$ be points of $x^{-1}(A)$ with $0 \leq t_{2}-t_{1} \leq \tau$. We will complete the proof by showing that $\left\|x\left(t_{2}\right)-x\left(t_{1}\right)\right\| \leq \delta$. If we define

$$
J=\left\{t>t_{1}: x(s) \in \mathbb{B}_{\varepsilon}(A) \text { for all } s \in\left[t_{1}, t\right]\right\},
$$

it follows that

$$
\left\|x(t)-x\left(t_{1}\right)\right\| \leq \int_{t_{1}}^{t} m(s) d s \leq \int_{t_{1}}^{t_{1}+\tau} m(s) d s \leq \delta
$$

for all $t$ in $J$ with $t \leq t_{1}+\tau$. Since $\delta<\varepsilon, t_{1}+\tau$ belongs to $J$, whence $\left\|x\left(t_{2}\right)-x\left(t_{1}\right)\right\| \leq \delta$.

In the following, we combine Proposition 3 with Theorems 8 and 9 to derive results on the asymptotic behaviour of solutions of (21).

Theorem 10. Let $G$ be a nonempty closed subset of $\mathbb{R}^{N}$, and let $g: G \rightarrow \mathbb{R}$ be a function. Assume that each $\xi$ in $G$ for which $g(\xi) \neq 0$ has a neighbourhood $U$ such that (17) holds and $f$ belongs to $\mathcal{F}(U \cap G)$. If $x: \mathbb{R}_{+} \rightarrow \mathbb{R}^{N}$ is a global solution of (21) with $x\left(\mathbb{R}_{+}\right) \subset G$ and $g \circ x$ is weakly meagre, then statements (a)-(d) of Theorem 8 hold.

Proof. Let $\xi$ in $G$ be such that $g(\xi) \neq 0$. By the hypotheses, there exists a neighbourhood $U$ of $\xi$ such that (17) holds and $f$ belongs to $\mathcal{F}(U \cap G)$. Let 
$\varepsilon>0$ be sufficiently small so that $\mathbb{B}_{2 \varepsilon}(\xi)$ lies in $U$. Then, setting $A=\mathbb{B}_{\varepsilon}(\xi)$, we see that $f$ is in the class $\mathcal{F}\left(\mathbb{B}_{\varepsilon}(A) \cap G\right)$. By Proposition 3, it follows that $x$ is uniformly continuous on $x^{-1}(A)$. An application of Theorem 8 completes the proof.

We remark that Theorem 10 contains a recent result by Teel [29, Theorem 1] as a special case. In the next theorem, it is assumed that $f$ is a member of $\mathcal{F}\left(\mathbb{B}_{\varepsilon}\left(g^{-1}(0)\right) \cap G\right)$ for some $\varepsilon>0$. Under the additional assumption that $g$ satisfies (20), it is then guaranteed that $x$ approaches $g^{-1}(0)$ (without positing the boundedness of $x$ ).

Theorem 11. Let $G$ be a nonempty closed subset of $\mathbb{R}^{N}$, and let $g: G \rightarrow \mathbb{R}$ be such that $g^{-1}(0)$ is closed and (20) holds for every nonempty closed subset $K$ of $G$. Assume that $f$ belongs to $\mathcal{F}\left(\mathbb{B}_{\varepsilon}\left(g^{-1}(0)\right) \cap G\right)$ ) for some $\varepsilon>0$. If $x: \mathbb{R}_{+} \rightarrow \mathbb{R}^{N}$ is a global solution of $(21)$ with $x\left(\mathbb{R}_{+}\right) \subset G$ and $g \circ x$ is weakly meagre, then statements (a)-(c) of Theorem 9 hold.

Proof. Fix $\delta$ in $(0, \varepsilon)$. By Proposition $3, x$ is uniformly continuous on the set $x^{-1}\left(\mathbb{B}_{\delta}\left(g^{-1}(0)\right)\right)$. An application of Theorem 9 completes the proof.

In the following we use Theorem 10 to obtain a version of a well-known result on $\omega$-limit sets of solutions of nonautonomous ordinary differential equations. For a nonempty open subset $D$ of $\mathbb{R}^{N}$ and a continuously differentiable function $V: \mathbb{R}_{+} \times D \rightarrow \mathbb{R}$, we define $V_{f}: \mathbb{R}_{+} \times D \rightarrow \mathbb{R}$ (the derivative of $V$ with respect to (21) in the sense that $(d / d t) V(t, x(t))=V_{f}(t, x(t))$ along a solution $x$ of $(21))$ by

$$
V_{f}(t, \xi)=\frac{\partial V}{\partial t}(t, \xi)+\sum_{i=1}^{N} \frac{\partial V}{\partial \xi_{i}}(t, \xi) f_{i}(t, \xi)
$$

for all $(t, \xi) \in \mathbb{R}_{+} \times D$, where $f_{1}, \ldots, f_{N}$ denote the components of $f$.

Corollary 4. Let $D$ be a nonempty open subset of $\mathbb{R}^{N}$, and let $V: \mathbb{R}_{+} \times D \rightarrow$ $\mathbb{R}$ be continuously differentiable. Assume that $V$ satisfies the following two conditions:

(a) for each $\xi$ in $\operatorname{cl}(D)$ there exists a neighbourhood $U$ of $\xi$ such that $V$ is bounded from below on the set $\mathbb{R}_{+} \times(U \cap D)$;

(b) there exists a lower semicontinuous continuous function $W: \operatorname{cl}(D) \rightarrow \mathbb{R}_{+}$ such that $V_{f}(t, \xi) \leq-W(\xi)$ for all $(t, \xi)$ in $\mathbb{R}_{+} \times D$.

Furthermore, assume that for every $\xi$ in $\operatorname{cl}(D)$ there exists a neighbourhood $U^{\prime}$ of $\xi$ such that $f$ belongs to $\mathcal{F}\left(U^{\prime} \cap D\right)$. Under these assumptions, if $x: \mathbb{R}_{+} \rightarrow$ $\mathbb{R}^{N}$ is a global solution of $(21)$ with $x\left(\mathbb{R}_{+}\right) \subset D$, then $\Omega(x) \subset W^{-1}(0)$.

Proof. If $\Omega(x)=\emptyset$ there is nothing to prove, so we assume that $\Omega(x) \neq \emptyset$. Since $(d / d t) V(t, x(t))=V_{f}(t, x(t))$ for all $t$ in $\mathbb{R}_{+}$, it follows from assumption (b) that the function $t \mapsto V(t, x(t))$ is nonincreasing, showing that the limit $l$ of $V(t, x(t))$ as $t \rightarrow \infty$ exists, where possibly $l=-\infty$. Let $\xi \in \Omega(x) \subset \operatorname{cl}(D)$. 
Then there exists a nondecreasing unbounded sequence $\left(t_{n}\right)$ in $\mathbb{R}_{+}$such that $\lim _{n \rightarrow \infty} x\left(t_{n}\right)=\xi$. By assumption (a) there exists a neighbourhood $U$ of $\xi$ such that $V$ is bounded from below on $\mathbb{R}_{+} \times(U \cap D)$. Now $x\left(\mathbb{R}_{+}\right) \subset D$, so there exists $n_{0}$ such that $x\left(t_{n}\right) \in U \cap D$ whenever $n \geq n_{0}$. Consequently, the nonincreasing sequence $\left(V\left(t_{n}, x\left(t_{n}\right)\right)\right)$ is bounded from below, showing that $l>-\infty$. Therefore

$$
\begin{aligned}
0 \leq \int_{0}^{\infty}(W \circ x)(t) d t \leq & -\int_{0}^{\infty} V_{f}(t, x(t)) d t \\
& =-\int_{0}^{\infty}(d / d t) V(t, x(t)) d t=V\left(0, x^{0}\right)-l<\infty,
\end{aligned}
$$

verifying that $W \circ x$ is in $L^{1}$, hence is weakly meagre. By assumption, for each $\xi$ in $\operatorname{cl}(D)$ there exists an open neighbourhood $U^{\prime}$ of $\xi$ such that $f$ belongs to $\mathcal{F}\left(U^{\prime} \cap D\right)$, implying that $f$ is in $\mathcal{F}\left(U^{\prime} \cap \operatorname{cl}(D)\right)$. Therefore, an application of Theorem 10 with $G=\operatorname{cl}(D)$ and $g=W$ establishes the claim.

Corollary 4 is essentially due to LaSalle [17] (see also [14, Satz 6.2, p. 140]). However, we point out that the assumption imposed on $f$ in Corollary 4 is weaker then that in [14] and [17], wherein it is required that, for every $\xi$ in $\operatorname{cl}(D)$, there exists a neighbourhood $U^{\prime}$ of $\xi$ such that $f$ is bounded on the set $\mathbb{R}_{+} \times\left(U^{\prime} \cap D\right)$. Furthermore, we impose only lower semicontinuity on the function $W$ (in contrast to [14] and [17], wherein continuity of $W$ is assumed).

The next result is a consequence of Theorem 11. It shows, in particular, that under a mild assumption on $f$ every global $L^{p}$-solution of (21) converges to zero.

Corollary 5. Assume that there exists $\varepsilon>0$ such that $f$ belongs to $\mathcal{F}\left(\mathbb{B}_{\varepsilon}(0)\right)$, and let $x: \mathbb{R}_{+} \rightarrow \mathbb{R}^{N}$ be a global solution of (21). Then the following statements hold:

(a) If $\|x(\cdot)\|$ is weakly meagre, then $\lim _{t \rightarrow \infty} x(t)=0$.

(b) If $x$ belongs to $L^{p}$ for some $p$ in $(0, \infty)$, then $\lim _{t \rightarrow \infty} x(t)=0$.

Proof. If $\|x(\cdot)\|$ is weakly meagre, then an application of Theorem 11 with $G=\mathbb{R}^{N}$ and $g=\|\cdot\|$ shows that $\lim _{t \rightarrow \infty} x(t)=0$. This establishes statement (a). To prove statement (b), let $x$ be in $L^{p}$ for some $p$ in $(0, \infty)$. Then, by Proposition 2(a), the function $\|x(\cdot)\|$ is meagre and hence is weakly meagre. By part (a) of the present result, $\lim _{t \rightarrow \infty} x(t)=0$.

Obviously, if (21) is autonomous (i.e., the differential equation in (21) has the form $\dot{x}(t)=f(x(t)))$, then the assumption that $f$ belongs to $\mathcal{F}\left(\mathbb{B}_{\varepsilon}(0)\right)$ for some $\varepsilon>0$ is trivially satisfied. Thus we may conclude that every weakly meagre global solution $t \mapsto x(t)$ of an autonomous ordinary differential equation converges to 0 as $t \rightarrow \infty$. 


\section{Autonomous differential inclusions}

In Section 5, we investigated the behaviour of systems within the framework of ordinary differential equations with Carathéodory righthand sides. However, as already alluded to in the Introduction, there are many meaningful situations wherein this framework is inadequate for purposes of analysis of dynamic behaviour. A prototypical example is that of a mechanical system with Coulomb friction which, formally, yields a differential equation with discontinuous right-hand side. Other examples permeate control theory and applications: a canonical case is a discontinuous feedback strategy associated with an on-off or switching device. Reiterating earlier comments, such discontinuous phenomena can be handled mathematically by embedding the discontinuities in set-valued maps, giving rise to the study of differential inclusions. The next goal is to extend our investigations on ordinary differential equations in this direction. Recall that $\mathcal{U}$ denotes the class of set-valued maps $\xi \mapsto F(\xi) \subset \mathbb{R}^{N}$, defined on $\mathbb{R}^{N}$, that are upper semicontinuous at each $\xi$ in $\mathbb{R}^{N}$ and take nonempty, convex, and compact values. The object of our study is the initial-value problem (10) for an autonomous differential inclusion, viz.

$$
\dot{x}(t) \in F(x(t)), \quad x(0)=x^{0} \in \mathbb{R}^{N}, \quad F \in \mathcal{U} .
$$

Recall that, by Theorem 4, for each $x^{0} \in \mathbb{R}^{N},(22)$ has at least one solution and every solution can be extended to a maximal solution.

The following proposition shows that, under suitable local boundedness assumptions on $F$, the solutions of (22) satisfy the uniform continuity assumptions required for an application of Theorems 8 and 9 . For a subset $A$ of $\mathbb{R}^{N}$ and for a member $F$ of $\mathcal{U}$ we denote (in a slight abuse of notation) the set $\cup_{a \in A} F(a)$ by $F(A)$.

Proposition 4. Let $A$ and $B$ be subsets of $\mathbb{R}^{N}$. Assume that $F\left(\mathbb{B}_{\varepsilon}(A) \cap B\right)$ is bounded for some $\varepsilon>0$ and that $x: \mathbb{R}_{+} \rightarrow \mathbb{R}^{N}$ is a solution of (22) with $x\left(\mathbb{R}_{+}\right) \subset B$. Then $x$ is uniformly continuous on $x^{-1}(A)$.

Proof. If $x^{-1}(A)=\emptyset$, then the assertion holds trivially. Assume that $x^{-1}(A) \neq$ $\emptyset$, and let $\delta$ in $(0, \varepsilon)$ be arbitrary. Define $\theta=\sup \left\{\|v\|: v \in F\left(\mathbb{B}_{\varepsilon}(A) \cap B\right)\right\}$, and let $\tau>0$ be sufficiently small so that $\tau \theta \leq \delta$. Adopting an argument similar to that used in the proof of Proposition 3 , it can be shown that $\left\|x\left(t_{2}\right)-x\left(t_{1}\right)\right\| \leq \delta$ for all $t_{1}$ and $t_{2}$ in $x^{-1}(A)$ with $0 \leq t_{2}-t_{1} \leq \tau$, proving that $x$ is uniformly continuous on $x^{-1}(A)$.

We now invoke Theorems 8 and 9 to derive counterparts of Theorems 10 and 11 for differential inclusions.

Theorem 12. Let $G$ be a nonempty closed subset of $\mathbb{R}^{N}$, let $g: G \rightarrow \mathbb{R}$ have the property that each $\xi$ in $G$ for which $g(\xi) \neq 0$ has a neighbourhood $U$ such that (17) holds. If $x: \mathbb{R}_{+} \rightarrow \mathbb{R}^{N}$ is a solution of (22) with $x\left(\mathbb{R}_{+}\right) \subset G$ and $g \circ x$ is weakly meagre, then statements (a) and (d) of Theorem 8 hold. Moreover, the following statements are true: 
$\left(\mathrm{b}^{\prime}\right)$ If $g^{-1}(0)$ is bounded and $\Omega(x) \neq \emptyset$, then $x$ is bounded and $x$ approaches the largest subset of $g^{-1}(0)$ that is weakly invariant with respect to (22).

$\left(\mathrm{c}^{\prime}\right)$ If $x$ is bounded, then $g^{-1}(0) \neq \emptyset$ and $x$ approaches the largest subset of $g^{-1}(0)$ that is weakly invariant with respect to (22).

Proof. Let $\xi$ in $G$ be such that $g(\xi) \neq 0$. By hypothesis, there exists $\varepsilon>0$ such that (17) holds with $U=\mathbb{B}_{\varepsilon}(\xi)$. By the upper semicontinuity of $F$, together with the compactness of its values, $F\left(\mathbb{B}_{\varepsilon}(U) \cap G\right)$ is bounded (see [2, Proposition 3, p. 42]. By Proposition 4, $x$ is uniformly continuous on $x^{-1}(U)$. Therefore, the hypotheses of Theorem 8 are satisfied, so statements (a)-(d) thereof hold. Combining statements (b) and (c) of Theorem 8 with the weak invariance of $\Omega(x)$ yields statements $\left(\mathrm{b}^{\prime}\right)$ and $\left(\mathrm{c}^{\prime}\right)$.

Theorem 13. Let $G$ be a nonempty closed subset of $\mathbb{R}^{N}$, let $g: G \rightarrow \mathbb{R}$ be such that $g^{-1}(0)$ is closed and (20) holds for every nonempty closed subset $K$ of $G$. Assume that $F\left(\mathbb{B}_{\varepsilon}\left(g^{-1}(0)\right) \cap G\right)$ is bounded for some $\varepsilon>0$. If $x: \mathbb{R}_{+} \rightarrow \mathbb{R}^{N}$ is a global solution of $(21)$ with $x\left(\mathbb{R}_{+}\right) \subset G$ and $g \circ x$ is weakly meagre, then statements (a) and (c) of Theorem 9 hold. Moreover, the following also holds:

$\left(\mathrm{b}^{\prime}\right)$ If $g^{-1}(0)$ is bounded, then $x$ is bounded and $x$ approaches the largest subset of $g^{-1}(0)$ that is weakly invariant with respect to (22).

Proof. Fix $\delta$ in $(0, \varepsilon)$. By Proposition $4, x$ is uniformly continuous on the set $x^{-1}\left(\mathbb{B}_{\delta}\left(g^{-1}(0)\right)\right)$. It follows immediately from Theorem 9 that statements (a)-(c) thereof hold. Assuming that $g^{-1}(0)$ is bounded, a combination of statements (b) of Theorem 9 with the weak invariance of $\Omega(x)$ yields statement $\left(\mathrm{b}^{\prime}\right)$.

If there exists a locally Lipschitz function $f: \mathbb{R}^{N} \rightarrow \mathbb{R}^{N}$ such that $F(x)=$ $\{f(x)\}$ (in this case, the differential inclusion (22) "collapses" to an autonomous differential equation which, for every $x^{0} \in \mathbb{R}^{N}$, has a unique solution satisfying $\left.x(0)=x^{0}\right)$, then the conclusions of Theorems 12 and 13 remain true when every occurence of "weakly invariant" is replaced with "invariant". We mention that precursors of Theorems 12 and 13 have appeared in [11] and [25].

Next, we exploit Theorem 13 to generalize LaSalle's invariance principle (see Corollary 3) to differential inclusions.

Corollary 6. Let $D$ be a nonempty open subset of $\mathbb{R}^{N}$, let $V: D \rightarrow \mathbb{R}$ be continuously differentiable, and set $V_{F}(\xi)=\max _{y \in F(\xi)}\langle\nabla V(\xi), y\rangle$ for all $\xi$ in $D$. Let $x: \mathbb{R}_{+} \rightarrow \mathbb{R}^{N}$ be a solution of (22) and assume that there exists a compact subset $G$ of $\mathbb{R}^{N}$ such that $x\left(\mathbb{R}_{+}\right) \subset G \subset D$. If $V_{F}(\xi) \leq 0$ for all $\xi$ in $G$, then $x$ approaches the largest subset of $V_{F}^{-1}(0) \cap G$ that is weakly invariant with respect to (22).

Proof. For later convenience, we first show that the function $V_{F}: D \rightarrow \mathbb{R}$ is upper semicontinuous. Let $\left(\xi_{n}\right)$ be a convergent sequence in $D$ with limit $\xi$ 
in $D$. Define $l=\limsup _{n \rightarrow \infty} V_{F}\left(\xi_{n}\right)$. From $\left(V_{F}\left(\xi_{n}\right)\right)$ extract a subsequence $\left(V_{F}\left(\xi_{n_{k}}\right)\right)$ with $V_{F}\left(\xi_{n_{k}}\right) \rightarrow l$ as $k \rightarrow \infty$. For each $k$, let $y_{k}$ be a maximizer of the continuous function $y \mapsto\left\langle\nabla V\left(\xi_{n_{k}}\right), y\right\rangle$ over the compact set $F\left(\xi_{n_{k}}\right)$, so $V_{F}\left(\xi_{n_{k}}\right)=\left\langle\nabla V\left(\xi_{n_{k}}\right), y_{k}\right\rangle$. Let $\varepsilon>0$ be arbitrary. By upper semicontinuity of $F, F\left(\xi_{n_{k}}\right) \subset \mathbb{B}_{\varepsilon}(F(\xi))$ for all sufficiently large $k$. Since $y_{k}$ lies in $F\left(\xi_{n_{k}}\right), F(\xi)$ is compact and $\varepsilon>0$ is arbitrary, we infer that $\left(y_{k}\right)$ has a subsequence (which we do not relabel) converging to a point $y^{*}$ in $F(\xi)$. Therefore,

$$
\begin{aligned}
\limsup _{n \rightarrow \infty} V_{F}\left(\xi_{n}\right)=l=\lim _{k \rightarrow \infty} V_{F}\left(\xi_{n_{k}}\right)=\lim _{k \rightarrow \infty}\left\langle\nabla V\left(\xi_{n_{k}}\right), y_{k}\right\rangle \\
=\left\langle\nabla V(\xi), y^{*}\right\rangle \leq V_{F}(\xi),
\end{aligned}
$$

confirming that $V_{F}$ is upper semicontinuous.

Evidently,

$$
\frac{d}{d t} V(x(t))=\langle\nabla V(x(t)), \dot{x}(t)\rangle \leq V_{F}(x(t)) \leq 0
$$

for almost every $t$ in $\mathbb{R}_{+}$, which leads to

$$
V(x(t))-V(x(0)) \leq \int_{0}^{t} V_{F}(x(s)) d s \leq 0
$$

for all $t$ in $\mathbb{R}_{+}$. Since $x$ is bounded, we conclude that the function $t \mapsto$ $\int_{0}^{t} V_{F}(x(s)) d s$ is bounded from below. But this function is also nonincreasing (because $V_{F} \leq 0$ on $G$ ), which ensures that $\lim _{t \rightarrow \infty} \int_{0}^{t} V_{F}(x(s)) d s$ exists and is finite. Consequently, $V_{F} \circ x$ is an $L^{1}$-function, showing that $V_{F} \circ x$ is weakly meagre. Since $V_{F}$ is upper semicontinuous and $V_{F} \leq 0$ on $G$, the function $G \rightarrow \mathbb{R}$ given by $\xi \mapsto\left|V_{F}(\xi)\right|$ is lower semicontinuous. Therefore, each $\xi$ in $G$ with $V_{F}(\xi) \neq 0$ has a neighbourhood $U$ such that $\inf \left\{\left|V_{F}(w)\right|: w \in G \cap U\right\}>0$. By statement $\left(\mathrm{c}^{\prime}\right)$ of Theorem 12 (with $\left.g=\left.V_{F}\right|_{G}\right)$ it follows that $x$ approaches the largest subset of $V_{F}^{-1}(0) \cap G$ that is weakly invariant with respect to $(22)$.

In Corollary 6, it is assumed that the solution $x$ is global (that is, defined on $\left.\mathbb{R}_{+}\right)$and has trajectory in some compact subset $G$ of $D$. These assumptions may be removed at the expense of strengthening the conditions on $V$ by assuming that its sublevel sets are bounded and that $V_{F}(\xi) \leq 0$ for all $\xi$ in $D$.

Corollary 7. Let $D, V, F$, and $V_{F}$ be as in Corollary 6. Assume that the sublevel sets of $V$ are bounded and that $V_{F}(\xi) \leq 0$ for all $\xi$ in $D$. If $x$ : $\left[0, \omega_{x}\right) \rightarrow \mathbb{R}^{N}$ is a maximal solution of (22) such that $\operatorname{cl}\left(x\left(\left[0, \omega_{x}\right)\right)\right) \subset D$, then $x$ is bounded, $\omega_{x}=\infty$, and $x$ approaches the largest subset of $V_{F}^{-1}(0)$ that is weakly invariant with respect to (22).

Proof. Since $(d / d t) V(x(t))=V_{F}(x(t)) \leq 0$ for almost all $t$ in $\left[0, \omega_{x}\right)$, we have the counterpart of $(23): V(x(t)) \leq V(x(0))$ for all $t$ in $\left[0, \omega_{x}\right)$. Since the sublevel sets of $V$ are bounded, it follows that $x$ is bounded. By assertion (b) of Lemma $2, \omega_{x}=\infty$. An application of Corollary 6 , with $G=\operatorname{cl}\left(x\left(\mathbb{R}_{+}\right)\right)$, completes the proof. 
Example 4. In this example we describe a typical application of Corollary 7. In part (a) of the example we analyze a general class of second-order differential inclusions; in part (b) we discuss a special case, a mechanical system subject to friction of Coulomb type.

(a) Let $k: \mathbb{R} \rightarrow \mathbb{R}$ be continuous with the property

$$
\lim _{|p| \rightarrow \infty} \int_{0}^{p} k=\infty
$$

Let $(p, v) \mapsto C(p, v) \subset \mathbb{R}$ be upper semicontinuous with nonempty, convex, compact values and with the property that, for all $(p, v)$ in $\mathbb{R}^{2}$,

$$
C_{*}(p, v):=\max \{v w: w \in C(p, v)\} \leq 0 .
$$

Consider the system

$$
\ddot{y}(t)+k(y(t)) \in C(y(t), \dot{y}(t)), \quad(y(0), \dot{y}(0))=\left(p^{0}, v^{0}\right) \in \mathbb{R}^{2} .
$$

Setting $x(t)=(y(t), \dot{y}(t))$, the second-order initial-value problem (26) can be expressed in the equivalent form

$$
\dot{x}(t) \in F(x(t)), \quad x(0)=x^{0}=\left(p^{0}, v^{0}\right) \in \mathbb{R}^{2},
$$

where the set-valued map $F \in \mathcal{U}$ is given by

$$
F(p, v)=\{v\} \times\{-k(p)+w: w \in C(p, v)\} .
$$

By Theorem 4, (27) has a solution and every solution can be extended to a maximal solution; moreover, every bounded maximal solution has interval of existence $\mathbb{R}_{+}$.

Claim A. For each $x^{0}=\left(p^{0}, v^{0}\right)$ in $\mathbb{R}^{2}$, every maximal solution $x=(y, \dot{y})$ of (27) is bounded (hence, has interval of existence $\mathbb{R}_{+}$) and approaches the largest subset $E$ of $C_{*}^{-1}(0)$ that is weakly invariant with respect to (27).

To establish this claim, we define $V: \mathbb{R}^{2} \rightarrow \mathbb{R}$ by

$$
V(p, v)=\int_{0}^{p} k(s) d s+v^{2} / 2 .
$$

Observe that, by property (24) of $k, V$ is such that, for every sequence $\left(\xi_{n}\right)$ in $\mathbb{R}^{2}, V\left(\xi_{n}\right) \rightarrow \infty$ as $n \rightarrow \infty$ and, as a result, every sublevel set of $V$ is bounded. Moreover,

$$
V_{F}(p, v)=\max _{\theta \in F(p, v)}\langle\nabla V(p, v), \theta\rangle=C_{*}(p, v) \leq 0 \quad \text { for all }(p, v) \in \mathbb{R}^{2} .
$$

Let $x^{0}=\left(p^{0}, v^{0}\right)$ be a point in $\mathbb{R}^{2}$ and let $x=(y, \dot{y})$ be a maximal solution of (27). An application of Corollary 7 , with $D=\mathbb{R}^{2}$, completes the proof of 
Claim A.

(b) As a particular example, consider a mechanical system wherein a mass is subject to a restoring force $k$ friction force of Coulomb type: the system can be written formally as

$$
\ddot{y}(t)+\operatorname{sgn}(\dot{y}(t))+k(y(t))=0 .
$$

Again, we assume that $k$ is continuous with property (24). This system may be embedded in the differential inclusion (26) with the set-valued map $C$ given by

$$
C(p, v):= \begin{cases}\{-1\}, & v>0 \\ {[-1,1],} & v=0 \\ \{+1\}, & v<0 .\end{cases}
$$

Claim B. For each $x^{0}=\left(p^{0}, v^{0}\right)$ in $\mathbb{R}^{2}$, every maximal solution $x=(y, \dot{y})$ of (27) (with $F$ and $C$ given by (28) and (29)) is bounded and approaches the set $k^{-1}([-1,1]) \times\{0\}$.

To prove this claim, we first note that in this case the function $C_{*}$ (defined in $(25))$ is given by

$$
C_{*}(p, v)=-|v| \leq 0 .
$$

Therefore, $C_{*}^{-1}(0)=\mathbb{R} \times\{0\}$. By Claim $\mathrm{A}$, for each $x^{0}=\left(p^{0}, v^{0}\right)$ in $\mathbb{R}^{2}$, every maximal solution $x=(y, \dot{y})$ of $(27)$ is bounded, is defined on $\mathbb{R}_{+}$, and approaches the largest subset $E$ of $\mathbb{R} \times\{0\}$ that is weakly invariant with respect to (27) (equivalently, (26)). Clearly $(0,0) \in E$ and so $E$ is non-empty. To conclude Claim B, it suffices to show that $E \subset k^{-1}([-1,1]) \times\{0\}$. Let $\left(p^{1}, 0\right) \in E$ be arbitrary. By weak invariance of $E$, there exists a solution $(z, \dot{z}): \mathbb{R}_{+} \rightarrow \mathbb{R}^{2}$ of $\ddot{z}+k(z(t)) \in C(z(t), \dot{z}(t))$, with $(z(0), \dot{z}(0))=\left(p^{1}, 0\right)$, such that $(z(t), \dot{z}(t)) \in E \subset \mathbb{R} \times\{0\}$ for all $t \in \mathbb{R}_{+}$. Therefore, for all $t \in \mathbb{R}_{+}$, $z(t)=p^{1}$ and $\dot{z}(t)=0=\ddot{z}(t)$. By the differential inclusion, it follows that $k\left(p^{1}\right) \in C\left(p^{1}, 0\right)=[-1,1]$ and so $p^{1} \in k^{-1}([-1,1])$. This completes the proof of Claim B.

\section{References}

1. H. Amann, Ordinary Differential Equations: An Introduction to Nonlinear Analysis, de Gruyter, Berlin, 1990

2. J. P. Aubin and A. Cellina, Differential Inclusions, Springer-Verlag, Berlin, 1984.

3. I. Barbălat, Systèmes d'équations différentielles d'oscillations non linéaires, Revue de Mathématiques Pures et Appliquées IV (1959) 267-270.

4. G.D. Birkhoff, Dynamical Systems, American Mathematical Society, Colloquium Publications Vol 9, Providence, 1927.

5. C.I. Byrnes and C.F. Martin, An integral-invariance principle for nonlinear systems, IEEE Trans. Automatic Control AC-40 (1995) 983-994.

6. F. H. Clarke, Optimization and Nonsmooth Analysis, Wiley, New York, 1983. 
7. F. H. Clarke, Yu. S. Ledyaev, R. J. Stern and P. R. Wolenski, Nonsmooth Analysis and Control Theory, Springer-Verlag, New York, 1998.

8. E. A. Coddington and N. Levinson, Theory of Ordinary Differential Equations, McGraw-Hill, New York, 1955.

9. C. Corduneanu, Integral Equations and Stability of Feedback Systems, Academic Press, New York, 1973.

10. K. Deimling, Multivalued Differential Equations, Walter de Gruyter, Berlin, 1992.

11. W. Desch, H. Logemann, E.P. Ryan, and E.D. Sontag, Meagre functions and asymptotic behaviour of dynamical systems, Nonlinear Analysis: Theory, Methods \& Applications 44 (2001) 1087-1109.

12. A.F. Filippov, Differential Equations with Discontinuous Righthand Sides, Kluwer, Dordrecht, 1988.

13. A. T. Fuller, The General Problem of the Stability of Motion (A.M. Lyapunov) (translation), Int. J. Control, bf 55 (1992), 531-773.

14. H.W. Knobloch and F. Kappel, Gewöhnliche Differentialgleichungen, B.G. Teubner, Stuttgart, 1974.

15. J. P. LaSalle, The extent of asymptotic stability, Proc. Nat. Acad. Sci. USA 46 (1960) 363-365.

16. - Some extensions of Liapunov's Second Method, IRE Trans. Circuit Theory CT-7 (1960) 520-527.

17. - Stability theory for ordinary differential equations, J. Differential Equations 4 (1968) 57-65.

18. — The Stability of Dynamical Systems, SIAM, Philadelphia, 1976.

19. H. Logemann and E. P. Ryan, Non-autonomous systems: asymptotic behaviour and weak invariance principles, J. Differential Equations 189 (2003) 440-460.

20. H. Logemann and E. P. Ryan, Asymptotic Behaviour of Nonlinear Systems, American Mathematical Monthly, 111 (2004), 864-889.

21. A. M. Lyapunov, Problème général de la stabilité du mouvement, Ann. Fac. Sci. Toulouse 9 (1907), 203-474. Reprinted in Ann. Math. Study No. 17, 1949, Princeton University Press.

22. A. M. Lyapunov, The general problem of the stability of motion (translator: A. T. Fuller), Int. J. Control 55 (1992) 531-773.

23. V. M. Popov, Hyperstability of Control Systems, Springer-Verlag, Berlin, 1973.

24. E. P. Ryan, Discontinuous feedback and universal adaptive stabilization, in Control of Uncertain Systems, (D. Hinrichsen and B. Mårtensson, eds.), Birkhäuser, Boston, 1990, pp. 245-258.

25. - - An integral invariance principle for differential inclusions with application in adaptive control, SIAM J. Control \& Optim. 36 (1998) 960-980.

26. S. Sastry, Nonlinear Systems: Analysis, Stability and Control, Springer-Verlag, New York, 1999.

27. G. V. Smirnov, Introduction to the Theory of Differential Inclusions, American Mathematical Society, Providence, 2002.

28. E. D. Sontag, Mathematical Control Theory, 2nd Edition, Springer, New York, 1998.

29. A. R. Teel, Asymptotic convergence from $L_{p}$ stability, IEEE Trans. Auto. Control AC-44 (1999) 2169-2170.

30. W. Walter, Ordinary Differential Equations, Springer-Verlag, New York, 1998. 